\title{
The amplification of large-scale motion in a supersonic concave turbulent boundary layer and its impact on the mean and statistical properties
}

\begin{tabular}{|r|l|}
\hline Journal: & Journal of Fluid Mechanics \\
\hline Manuscript ID & JFM-18-S-0754.R2 \\
\hline mss type: & JFM Papers \\
\hline Complete List of Authors: & $\begin{array}{l}\text { Wang, Qian-Cheng; National University of Defense Technology, } \\
\text { Wang, Zhenguo; National University of Defense Technology, } \\
\text { Sun, Mingbo; Science and Technology on Scramjet Lab } \\
\text { Yang, Rui; National University of Defense Technology, College of } \\
\text { Aerospace Science and Engineering } \\
\text { Zhao, Yuxin } \\
\text { Hu, Zhiwei; University of Southampton, SES-Aero }\end{array}$ \\
\hline Keyword: & $\begin{array}{l}\text { High-speed flow < Aerodynamics, Compressible boundary layers < } \\
\text { Compressible Flows, Boundary layer control < Boundary Layers }\end{array}$ \\
\hline \multicolumn{2}{|l}{} \\
\hline
\end{tabular}

\section{SCHOLARONE \\ Manuscripts}




\title{
The amplification of large-scale motion in a supersonic concave turbulent boundary layer and its impact on the mean and statistical properties
}

\author{
Qian-cheng Wang ${ }^{1}$, Zhen-guo Wang $^{1}$, Ming-bo Sun ${ }^{1} \dagger$, Rui Yang ${ }^{1}$, \\ Yu-xin $\mathrm{Zhao}^{1}$, and Zhiwei $\mathrm{Hu}^{2}$ \\ ${ }^{1}$ Science and Technology on Scramjet Laboratory, National University of Defense Technology, \\ Changsha, 410073, China, \\ ${ }^{2}$ Faculty of Engineering and the Environment, University of Southampton, Southampton SO17 \\ 1BJ, United Kingdom
}

(Received $\mathrm{xx}$; revised $\mathrm{xx}$; accepted $\mathrm{xx}$ )

Direct numerical simulation is conducted to uncover the response of a supersonic turbulent boundary layer to streamwise concave curvature and the related physical mechanisms at a Mach number of 2.95. Streamwise variations of mean flow properties, turbulent statistics and turbulent structures are analyzed. A method to define the boundary layer thickness based on the principal strain rate is proposed, which is applicable for boundary layer subjected to wall-normal pressure and velocity gradients. While the wall friction grows with the wall turning, the friction velocity decreases. A logarithmic region with constant slope exists in the concave boundary layer. However, with smaller slope, it is located lower than that of the flat boundary layer. Streamwise varying trends of the velocity and the principal strain rate within different wall-normal regions are different. The turbulent level is promoted by the concave curvature. Due to the increased turbulent generation in the outer layer, secondary bumps are noted in profiles of streamwise and spanwise turbulent intensity. Peak positions in profiles of wall-normal turbulent intensity and Reynolds shear stress are pushed outward because of the same reason. Attributed to the Görtler instability, the streamwise extended vortices within the hairpin packets are intensified and more vortices are generated. Through accumulations of these vortices with similar sense of rotation, large-scale streamwise roll cells are formed. Originated from the very large-scale motions and by promoting the ejection, sweep and spanwise events, the formation of large-scale streamwise roll cells is the physical cause of the alterations of mean properties and turbulent statistics. The roll cells further give rise to the vortex generation. The large amount of hairpin vortices formed in the near-wall region lead to the improved wall-normal correlation of turbulence in the concave boundary layer.

Key words: streamline concave curvature, turbulent boundary layer, supersonic, direct numerical simulation, VLSM

$\dagger$ Email address for correspondence: sunmingbonudt@sina.cn 


\section{Introduction}

Turbulence is frequently subjected to wall curvature, which influences the boundary layer development and turbulent structures. There are many situations of practical importance where a boundary layer is actually curved, such as on aerofoils and turbine blades, in aircraft inlets, combustion chambers and nozzles. Many studies have been carried out to investigate effects of the surface curvature for both incompressible and compressible flow in the past few decades (see e.g. Bradshaw 1974; Hoffmann et al. 1985; Donovan et al. 1994; Patel \& Sotiropoulos 1997; Humble et al. 2012; Tichenor et al. 2013; Tong et al. 2017). It is now well-known that the concave and the convex surface curvatures have opposite impacts on the boundary layer. The concave curvature destabilizes the boundary layer, while the convex stabilizes it.

One typical impact of the concave curvature on the boundary layer is to induce into the flow counter-rotating Görtler vortices, which promotes the boundary layer transition and strengthens turbulence levels (see e.g. Görtler 1954; Floryan 1991; Saric 1994; Roghelia et al. 2017). The generation of Görtler vortices on a concave wall is the result of centrifugal instability (or Görtler instability) which is brought by the imbalance between the centrifugal force and the wall-normal pressure gradient. Previous studies on the Görtler instability revealed that the formation and evolution of Görtler vortices highly depend on initial conditions and external disturbances (see e.g. Hall 1983; Wu et al. 2011; Dempsey et al. 2017) and it usually undergoes linear and nonlinear development (see e.g. Tandiono et al. 2008; Schrader et al. 2011). The secondary instability and the related breakup of Görtler vortices lead to the final transition of boundary layer (see e.g. Swearingen \& Blackwelder 1987; Ren \& Fu 2015; Wang et al. 2018).

Different from laminar flow, it is very difficult to identify Görtler vortices in turbulent boundary layer. However, the impact of Görtler instability remains. Early experiments conducted for impressible flow indicate that the Görtler instability could introduce largescale longitudinal roll cells into turbulent boundary layer, which are larger and more energetic than large eddies in a flat-plate boundary layer(Hoffmann et al. 1985; Barlow \& Johnston 1988a,b). Mixing across the boundary layer was enhanced by the roll cells bringing high-momentum fluid close to the wall and amplifying large-scale motions in the wall-normal direction. Barlow \& Johnston $(1988 a, b)$ pointed out that these structures did not have well defined cores of concentrated vorticity and they should not be defined as vortices under any reasonable definition of the term. Through flow visualization, Barlow \& Johnston (1988b) found that the large-scale roll cells did not have preferred spanwise locations and did not last long if the incoming boundary layer was relatively free of spanwise non-uniformities. By using small vortex generators to introduce weak vortices into the boundary layer, Barlow \& Johnston (1988a) found that the original disturbances within the boundary layer could be significantly amplified into stationary roll cells by the concave curvature. The inward flows suppress the bursting process, while the outward flow enhance it, through which the turbulent production is greatly promoted. MokhtarzadehDehghan \& Yuan (2002) gave similar findings. They also showed that the turbulence enhancement on concave walls is most pronounced in the outer $80 \%$ of the boundary layer. In addition to the enhancement of turbulent levels in a concave turbulent boundary layer, the wall friction and heat transfer coefficients are also increased relative to their canonical flat-plate values (see e.g. Smits \& Wood 1985; Ozalp \& Umur 2003).

In contrast to the flat-plate boundary layer which is subjected to the simple shear $\partial U / \partial y$, the curved compressible boundary layer is subjected to additional combined effects of streamline curvature $\partial V / \partial x$, pressure gradients, $\partial p / \partial x, \partial p / \partial y$, and bulk compression $\nabla \cdot \mathbf{U}$ (Bradshaw 1973, 1974). Although it has been known for some time 


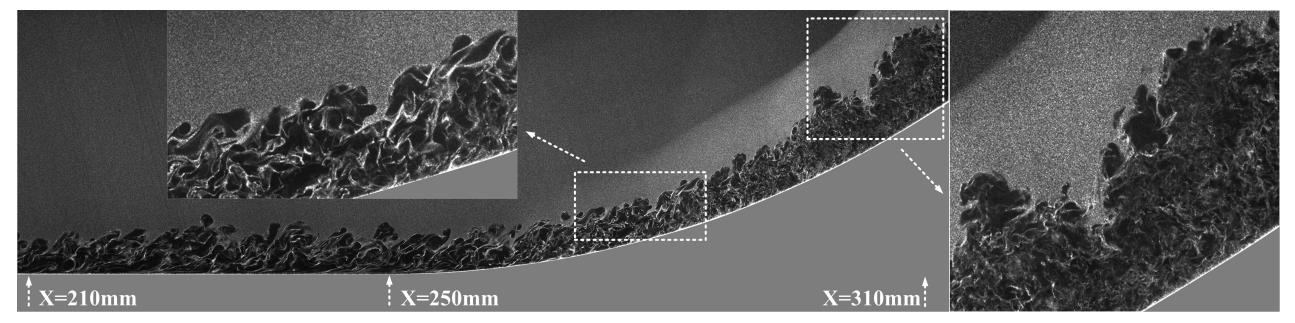

FIGURE 1. Instantaneous streamwise flow structures of a supersonic concave boundary layer.

(Wang \& Wang 2016)

that the concave curvature greatly affects the turbulence structures, further experimental and numerical investigations into this reorganization in the supersonic flow regime is still required to reveal detailed turbulent flow. So far, most of the studies regarding the supersonic concave boundary layer are focused on the mean and statistical properties (see e.g. Sturek \& Danberg 1972a,b; Laderman 1980; Smith \& Smits 1995).

Jayaram et al. (1987) measured the characteristics of the supersonic turbulent boundary layer formed on a concave wall and a compression ramp with Mach number 2.87. They found that both configurations increase the turbulent levels. Similar to the finding of Hoffmann et al. (1985) for incompressible flow, Jayaram et al. (1987) also found a dip below the logarithmic law in the velocity profile. However, different from the incompressible regime, they found no evidence for the presence of longitudinal roll cells. At Mach number 2.86, Donovan et al. (1994) found that the wall shear stress was significantly amplified by about $125 \%$ over the concave wall when comparing with the flat-plate case. The dip below the logarithmic region was not noticed in the concave region, while instead, it was noticed in the recovery region downstream of the concave wall. Similar phenomenon was also found by Smith \& Smits (1995). By measuring the boundary layer formed on a flat plate with similar pressure distribution to that of the concave wall, they suggested that the appearance of the dip might be linked directly to the presence of streamline curvature. Wang et al. (2016a) found that the principal strain rate had different streamwise varying trends at different wall-normal locations. Flaherty \& Austin (2013) examined the surface heat transfer of a hypersonic concave boundary layer with Mach number up to 7.45. They found significant augmentation in the surface heat transfer over the baseline flat plate. The heat transfer data are found to collapse when plotted versus local turning angle rather than downstream distance. Regarding the adverse pressure gradient(APG) brought by the streamwise concave curvature, previous studies showed that the streamwise APG had similar impact on the boundary layer to that of the concave curvature, which destabilizes boundary layers and promotes turbulence (Franko \& Lele 2014; Wang et al. 2016b). A frequently used parameter proposed by Clauser (1954) to evaluate the streamwise pressure gradient is $\beta=\delta^{*} / \tau_{w} \cdot \partial p / \partial x$ (where $\delta^{*}$ is the displacement thickness, $\tau_{w}$ is the wall shear stress, and $\partial p / \partial x$ is the streamwise pressure gradient). Franko \& Lele (2014) showed that for all transition mechanisms, the APG can increase the linear growth rates and accelerate the transition to turbulence. Experimental investigation conducted by Lewis et al. (1972) on an axisymmetric model without streamwise curvature revealed a good general agreement of the mean velocity profile with the Spalding law (Spalding 1961). Wang et al. (2016b) have similar findings in a supersonic APG flat-plate boundary layer.

Upon previous studies regarding the supersonic turbulent concave boundary layer, it is still unclear that how the turbulent structures are affected by the concave curvature. The alteration of turbulent structures is the physical cause of previously noticed changes in 
mean and statistical properties. With high-resolution flow visualization, Wang \& Wang (2016) clearly captured the streamwise change of turbulent structures from the flatplate to the concave region, and it is found that the large-scale structures tend to beak up into smaller ones in the concave region, as shown in figure 1. This partly explains the promotion of turbulent intensity. But with only two-dimensional visualizations of turbulent structures in a streamwise slice being presented, it is still difficult to find the relevant physical mechanisms. More recently, Tong et al. (2017) employs direct numerical simulation (DNS) to study the supersonic concave turbulent boundary layer. By using dynamic mode decomposition, they found spatial structures that looks similar to the longitudinal roll cells. But with a shock wave being introduced, the flow was complicated and physical mechanisms related to the streamline curvature are not recovered.

In the present investigation, aimed for a detailed understanding of the response of supersonic turbulent boundary layer to the streamwise concave curvature and to recover the related physical mechanisms, a DNS is conducted. Both the mean properties and the turbulent structures will be analyzed, and the related physical mechanisms will be revealed. The rest of the paper is organized as follows. In Section 2 the numerical method and the computational setup are introduced. In Section 3, responses of mean properties, turbulent statistics and turbulent structures to the curvature are presented. Discussions on the physical causes of the changes of mean properties in the concave boundary layer and their relations with instantaneous turbulent structures are given in Section 4.

\section{Simulation details}

\subsection{Numerical methods and inflow conditions}

The code used in present study has been applied to many studies of instability, transition and turbulence in supersonic flows (Sandham et al. 2014; Sandham 2016; Wang et al. 2015b; Sun et al. 2017). The details of the DNS methodology have been documented by Touber \& Sandham (2009); Touber (2010). Therefore, only a brief description is given here.

The three-dimensional compressible Navier-Stokes equations are solved directly without modeling. The working fluid is assumed to be a perfect gas with ratio of heat capacity $\gamma=1.4$. The coefficient of viscosity $\mu$ is computed according to the Sutherland's law. The coefficient of thermal conductivity $\kappa$ is computed from the relation $\kappa=\mu C_{p} / P r$, where $\operatorname{Pr}=0.71$ is the molecular Prandtl number and $C_{p}$ is the heat capacity at constant pressure.

The non-dimensionalized equations are solved by using an explicit 4th-order central spatial differencing scheme for the spatial derivatives. The 3rd-order explicit RungeKutta scheme is used for the time integration. The entropy splitting approach (Sandham et al. 2002; Yee et al. 2000) is used for the Euler terms and the Laplacian formulation is employed for the viscous terms. A digital-filter-based method (Xie \& Castro 2008) is used for the generation of turbulent inflow. The mean inflow profile is generated from the similarity solution of compressible boundary layer equation, as was used by Touber \& Sandham (2009). The inlet RMS values are generated by transforming the incompressible DNS data of Schlatter \& Orlu (2010) with a similar Reynolds number into the compressible.

Table 1 outlines the free-stream inflow conditions for the present simulation. The inflow parameters including the free-stream Mach-number $M_{\infty}$, density $\rho_{\infty}$ and temperature $T_{\infty}$ are prescribed in accordance with the Mach 2.95 experiments of Wang \& Wang (2016) conducted in the National University of Defense Technology. Throughout this 


$\begin{array}{lcccc}\text { Mach number } & \text { Free streamwise } & \text { Stagnation } & \text { Stagnation } & \text { BL thickness } \\ & \text { velocity } & \text { temperature } & \text { pressure } & \text { at the inlet } \\ M a_{\infty} & U_{\infty} & T_{\infty} & P_{\infty} & \delta_{i} \\ 2.95 & 606 \mathrm{~m} / \mathrm{s} & 288 \mathrm{~K} & 100 \mathrm{kPa} & 5.0 \mathrm{~mm}\end{array}$

TABLE 1. Inflow conditions for the Mach 2.95 DNS of the concave turbulent boundary-layer (BL)

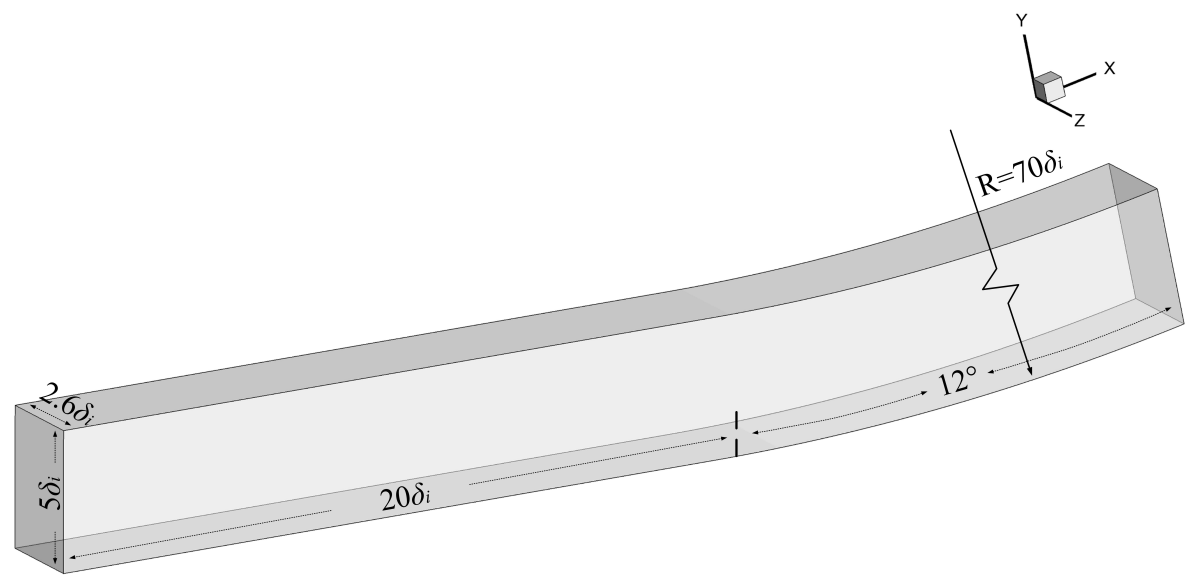

Figure 2. Computational domain for the DNS of concave turbulent boundary layer. The reference length $\delta_{i}$ is the boundary layer thickness based on $99 \%$ of the free-stream velocity at the inlet plane. $x, y$ and $z$ denote the streamwise, wall-normal and spanwise coordinates, respectively.

paper, subscripts " $\infty$ " and "w" are used to denote quantities at the boundary layer edge and at the wall, respectively.

\subsection{Computational domain and simulation set-up}

Figure 2 shows the computational domain for DNS of the concave turbulent boundary layer. Throughout the paper, the upper cases of $X$ and $Y$ are used to denote the Cartesian coordinates, and the lower cases of $x, y$ and $z$ are used to denote the streamwise direction along the wall, the wall-normal direction and the spanwise direction, respectively. In the following discussion, all of the Cartesian axial coordinate $X$, streamwise coordinate $x$ and wall-turning angle $\alpha$ may be used to denote the axial position on different occasions. To facilitate the comparison, streamwise evolutions of $x$ and $\alpha$ with $X$ are calculated, as shown in figure 3 . Because of the relatively small overall wall-turning, the streamwise coordinate $x$ is close to the Cartesian axial coordinate $X$ and the wall-turning angle grows almost linearly with $X$ in the concave region.

The use of digital filter to generate the inflow boundary layer condition reduces length for the boundary layer to be fully developed. According to the assessment given by Wang et al. (2015b), a distance of 12 times the inflow boundary-layer thickness is enough to obtain realistic mean and RMS profiles from the digital filter inflow generator. In present DNS study, a distance of $20 \delta_{i}$ to the inlet is set to have a fully developed turbulent boundary layer before wall turning. The radius of the concave wall is $R=350 \mathrm{~mm}$ and the overall turning angle is $\alpha=12^{\circ}$. The domain size and the grid resolution are summarized in table 2. Grid resolutions at both inlet and outlet of the computational domain are 

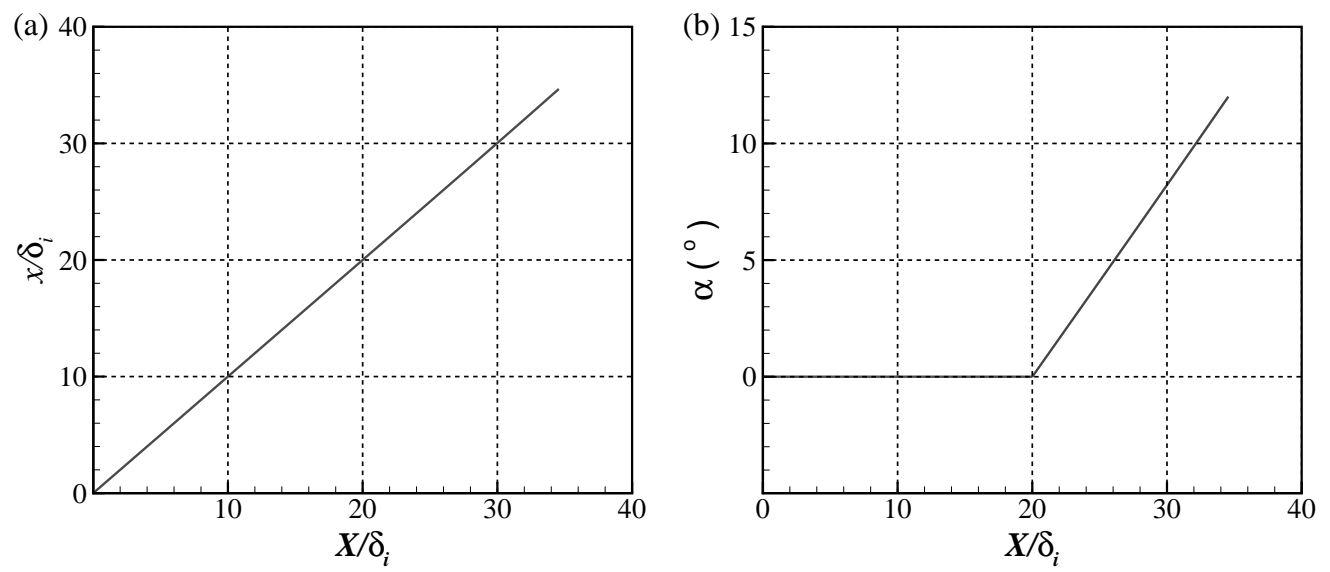

Figure 3. Streamwise evolutions of (a) streamwise coordinate $x$ and (b) wall-turning angle with Cartesian axial coordinate $X$.

$$
\begin{array}{ll}
N_{x} \times N_{y} \times N_{z} & 930 \times 240 \times 210 \\
\left(L_{x} / \delta_{i}\right) \times\left(L_{y} / \delta_{i}\right) \times\left(L_{z} / \delta_{i}\right) & 20.0 \times 5.0 \times 2.6 \\
\left(L_{y, \text { in }}^{+}\right) \times\left(L_{z, \text { in }}^{+}\right) & 1540 \times 800.8 \\
\left(L_{y, \text { out }}^{+}\right) \times\left(L_{z, \text { out }}^{+}\right) & 2800 \times 1456 \\
\left(\Delta x^{+} \times \Delta y_{\text {min }}^{+} \times \Delta z^{+}\right)_{\text {in }} & 6.2 \times 0.6 \times 3.8 \\
\left(\Delta x^{+} \times \Delta y_{\text {min }}^{+} \times \Delta z^{+}\right)_{\text {out }} & 11.8 \times 1.1 \times 7.0 \\
\Delta y_{\text {in }, \text { max }}^{+} & 6.0 \\
\Delta y_{\text {out }, \text { max }}^{+} & 9.6
\end{array}
$$

TABle 2. Domain size and grid resolution for the DNS. $L_{x}, L_{y}$ and $L_{z}$ are the streamwise, wall-normal and spanwise sizes of the computational domain, respectively. $\Delta x^{+}$and $\Delta z^{+}$are spacings of the uniformly distributed grid in the streamwise and spanwise directions, respectively. $\Delta y_{\min }^{+}$and $\Delta y_{\max }^{+}$are the minimum and maximum wall-normal spacings within the height of $0 \leqslant y / \delta_{i} \leqslant 1.2$. The subscripts 'in' and 'out' are used to denote the inlet and outlet of the computational domain, respectively. At the inlet, the grid spacings are normalized with the viscous length scale $y_{\tau}$ evaluated at the streamwise position of $x / \delta_{i}=18.3$.

presented. In the streamwise and spanwise directions, the grids are uniformly distributed. In the wall-normal direction, a stretched grid is used to have fine grids in the near-wall region. A stretching function in a hyperbolic tangent form (Vinokur 1980) is used. The distribution of grid size in the wall-normal direction is given in figure 4 . The superscript ' + ' denotes the normalization by the viscous length scale $y_{\tau}=\nu_{w} / u_{\tau} . \nu_{w}$ is the kinematic viscosity at the wall and $u_{\tau}=\sqrt{\tau_{w} / \rho_{w}}$ is the friction velocity, where $\tau_{w}$ is the wall shear stress and $\rho_{w}$ is the flow density at the wall. Though the dimensional size of the grid is kept unchanged in streamwise direction, due to the streamwise decrease of $u_{\tau}$ (we will find this in Section 3.1.2), the grid size with inner-scaling varies. Despite of the obvious larger inner-scaled grid size at the outlet of the computational domain than that at the inlet (see table 2), it is still fine enough to the resolve the wall-bounded turbulence in the concave region.

No-slip boundary condition is used for the bottom wall. The wall temperature is fixed 


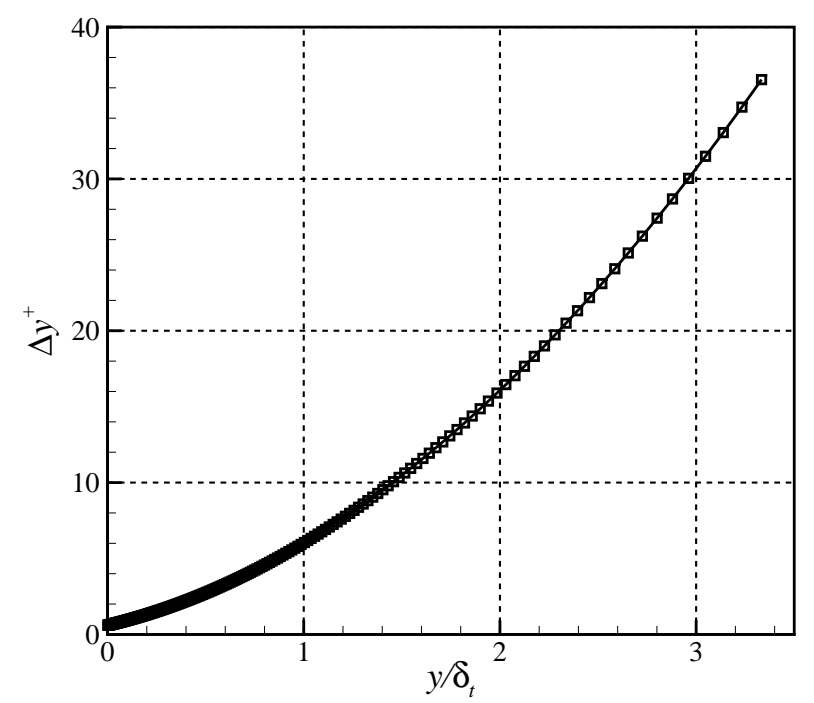

FiguRE 4. The wall-normal distribution of grid spacing

$$
\begin{array}{lccccccc}
R e_{\theta} & R e_{\tau} & \theta(\mathrm{mm}) & \delta^{*}(\mathrm{~mm}) & \delta_{0.99}(\mathrm{~mm}) & u_{\tau} / U_{\infty} & \operatorname{Re}_{\theta, v d} & \operatorname{Re}_{\delta^{*}, v d} \\
3410.7 & 361.4 & 0.42 & 2.28 & 5.92 & 0.05 & 1120.7 & 1722.2
\end{array}
$$

TABLE 3. Mean boundary layer parameters at the streamwise position of $x=18.3 \delta_{i}$.

to the free stream stagnation temperature. For the outflow and for the top boundary, characteristic boundary conditions are used. For two side boundaries, the periodic boundary condition is utilized.

\subsection{Validation of DNS data}

The mean boundary-layer parameters, and Reynolds numbers based on the momentum and displacement thicknesses at the the streamwise position of $x=18.3 \delta_{i}$ in the flat plate region are listed in table 3 , where $\theta$ is the momentum thickness, $\delta^{*}$ is the displacement thickness and $\delta_{0.99}$ is the boundary layer thickness based on the $99 \%$ criteria. Representative Reynolds numbers are calculated with relations of $R e_{\theta}=\rho_{\infty} U_{\infty} \theta / \mu_{\infty}$ and $R e_{\tau}=\rho_{w} u_{\tau} \delta_{0.99} / \mu_{w}$. Reynolds numbers based on the van Driest transformed momentum and displacement thicknesses are also shown in the table, where $R e_{\theta, v d}=\rho_{w} U_{\infty}^{v d} \theta^{v d} / \mu_{w}$ and $R e_{\delta^{*}, v d}=\rho_{w} U_{\infty}^{v d} \delta^{* v d} / \mu_{w}$.

For validation, the mean velocity and turbulent statistics given by Schlatter \& Orlu (2010) for an incompressible boundary layer, with Reynolds numbers $R e_{\tau}=359.4, R e_{\theta}=$ 1006.5 and $R e_{\delta^{*}}=1459.4$ which are similar to the van Driest transformed Reynolds numbers in the present investigation, are used for comparison, as shown in figure 5 (a) and (b). The results acquired by Guarini et al. (2000) with $R e_{\theta}=1577$ at Mach 2.5 and by Maeder et al. (2001) with $R e_{\theta}=3028$ at Mach 3.0 are also included in the figure.

The van Driest transformed mean-velocity profile at the streamwise position of $x=$ $18.3 \delta_{i}$ is presented in figure $5(\mathrm{a})$. In the figure, $U^{+}$is defined as 
(a)

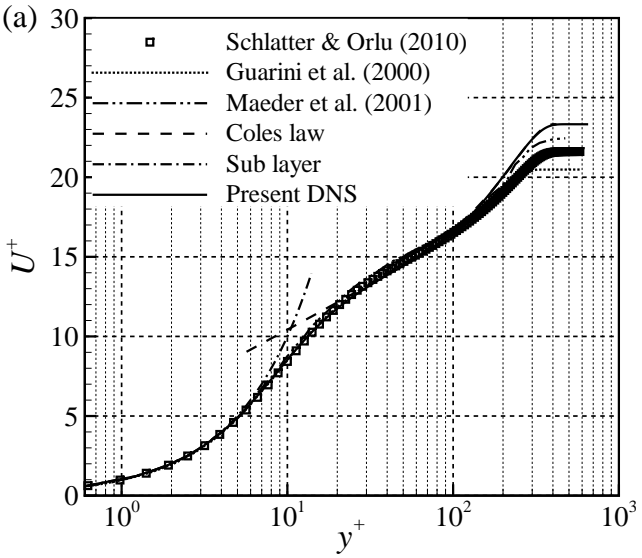

(c)

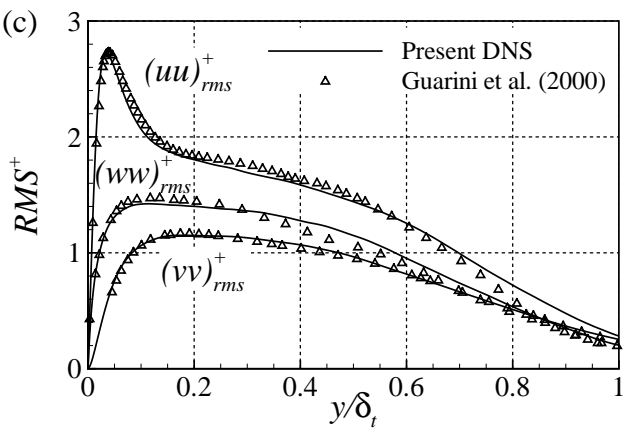

(b)

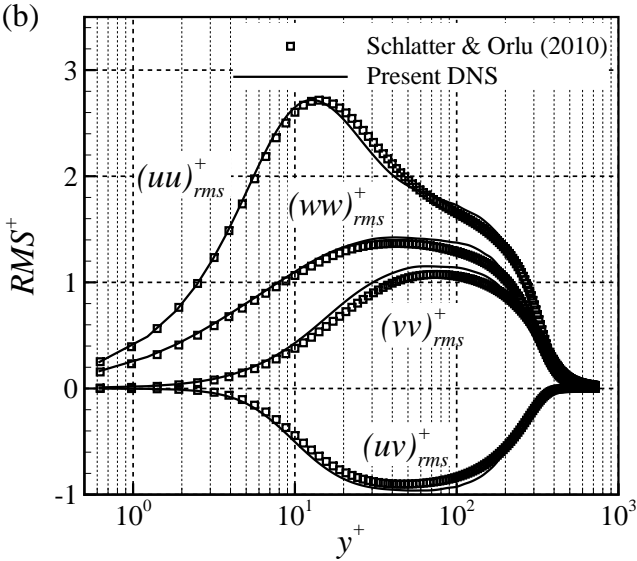

(d)

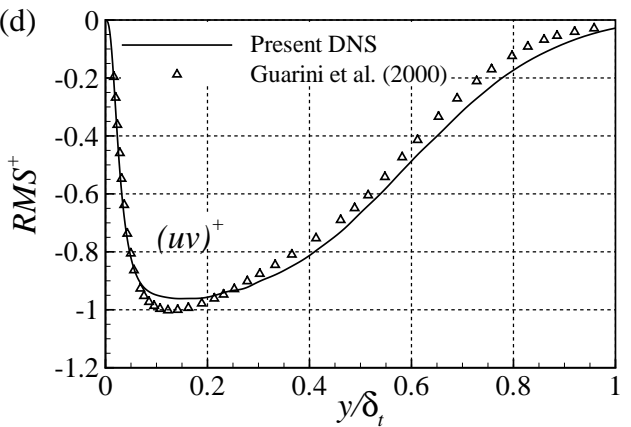

Figure 5. Profiles of mean velocity and r.m.s. values at the streamwise position of $x=18.3 \delta_{i}$ and comparisons with that of Schlatter \& Orlu (2010) and Guarini et al. (2000): (a) van Driest transformed mean velocity profile as well as its comparison with Coles law (White 2006); (b) profiles of turbulent statistics with compressible and inner scalings; (c) profiles scaled r.m.s. plotted against $y / \delta_{t} ;(\mathrm{d})$ profiles of $(u v)_{r m s}^{+}$plotted against $y / \delta_{t}$.

$$
U^{+}=1 / u_{\tau} \int_{0}^{U}\left(\overline{T_{w}} / \bar{T}\right)^{1 / 2} d U
$$

The van Driest transformed mean velocity agrees well with the law of the wall, as well as with those given by Schlatter \& Orlu (2010), Guarini et al. (2000) and Maeder et al. (2001). Profiles of root mean square (r.m.s.) of the streamwise and wall-normal velocity components scaled by $\sqrt{\bar{\rho} / \bar{\rho}_{w}} / u_{\tau}$ are given in figure 5 (b), (c), respectively, where $(u u)_{r m s}^{+}=\sqrt{\bar{\rho} / \bar{\rho}_{w}} \cdot \sqrt{\overline{u^{\prime 2}}} / u_{\tau}$ is the scaled r.m.s. of the streamwise velocity component, $(v v)_{r m s}^{+}=\sqrt{\bar{\rho} / \bar{\rho}_{w}} \cdot \sqrt{\overline{{v^{\prime}}^{2}}} / u_{\tau}$ is the scaled r.m.s. of the wall-normal velocity component, and $(w w)_{r m s}^{+}=\sqrt{\bar{\rho} / \bar{\rho}_{w}} \cdot \sqrt{\overline{w^{\prime 2}}} / u_{\tau}$ is the scaled r.m.s. of the spanwise velocity component. $u^{\prime}, v^{\prime}$ and $w^{\prime}$ are the fluctuations about the Reynolds averaged value. The profile of $\overline{u^{\prime} v^{\prime}}$ scaled by $\left(\bar{\rho} / \bar{\rho}_{w}\right) / u_{\tau}^{2}$ is given in figure $5(\mathrm{~d})$, where $(u v)^{+}=\bar{\rho} / \bar{\rho}_{w} \cdot \overline{u^{\prime} v^{\prime}} / u_{\tau}^{2}$. In figure 5 (b), the r.m.s. profiles are presented with inner scaling, and they compare well with the incompressible results of Schlatter \& Orlu (2010). In figure 5 (c) and (d), the same data are presented with outer scaling and they are also in good agreement with that of Guarini et al. (2000).

Spanwise profiles of two-point spatial correlation for the streamwise velocity component at six wall-normal positions, $y^{+}=5,10,20,50,100$ and 160 , in the flat-plate region are 
(a)

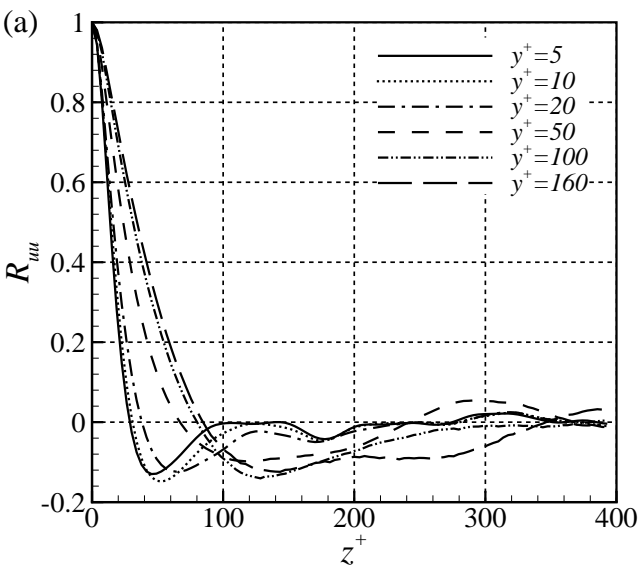

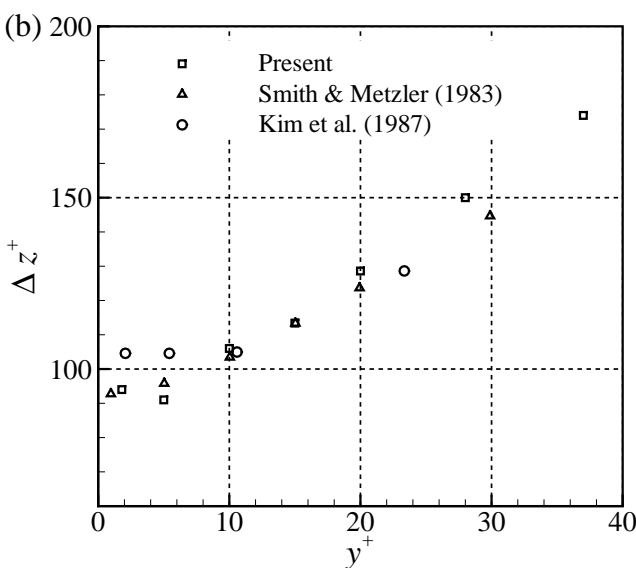

FiguRe 6. (a) Spanwise profiles of two-point spatial correlation at the streamwise position $x=18.3 \delta_{i}$. (b) Wall-normal variation of mean spanwise streak spacing with inner scaling and its comparison with the results of Smith \& Metzler (1983) and Kim et al. (1987).

given in figure 6(a). As can be evidently seen from the figure, the near-wall correlations (e.g. $y^{+}=5,10$ and 20) are close to zero for large separation. This indicates that the two-point correlation functions are sufficiently decorrelated over the distance of $L_{z} / 2$. For profiles away from the wall (e.g. $\left.y^{+}=160\right)$, the spanwise correlation brought by the large-scale coherence could still be clearly seen, which is similar to that given by Guarini et al. (2000). According to the locations of minimum value in the two-point correlation profiles, the variation of spacing of the low-speed streak with the wall-normal distance can be estimated, as shown in figure 6(b). The results of Smith \& Metzler (1983) and Kim et al. (1987) are included in the figure for comparison. Clearly, the results of present DNS agrees with those of Smith \& Metzler (1983) and Kim et al. (1987), thus ensuring that the computational domain in the spanwise direction is wide enough not to inhibit the turbulence dynamics.

The comparison of skin friction acquired at $x=18.3 \delta_{i}$ with those obtained by Pirozzoli et al. (2004), Guarini et al. (2000) and Maeder et al. (2001) is given in figure 7. For present DNS, the friction coefficient $C_{f}=\tau_{w} /\left(0.5 \rho_{\infty} U_{\infty}^{2}\right)$ is calculated to be 0.00185 . Following custom and to facilitate the comparison of data acquired at different Mach numbers, the friction coefficient and the Reynolds number are reduced to 'incompressible' values by employing the van Driest II transformation

$$
C_{f_{i n c}}=F_{c} C_{f}, R e_{\theta_{i n c}}=\frac{\mu_{\infty}}{\bar{\mu}_{w}} R e_{\theta}
$$

where, the subscript 'inc' denotes the incompressible value and for adiabatic wall

$$
F_{c}=\frac{\bar{T}_{w} / T_{\infty}-1}{\arcsin ^{2} \alpha}, \alpha=\frac{\bar{T}_{w} / T_{\infty}-1}{\sqrt{\bar{T}_{w} / T_{\infty}\left(\bar{T}_{w} / T_{\infty}-1\right)}}
$$

The transformed incompressible friction coefficient $C_{f_{i n c}}$ and Reynolds number $R e_{\theta_{i n c}}$ for present DNS are 0.0038 and 1696, respectively. The incompressible skin friction laws of Kámán-Schoenherr and Blasisus (Hopkins \& Inouye 1971) are also included in the figure for comparison. 


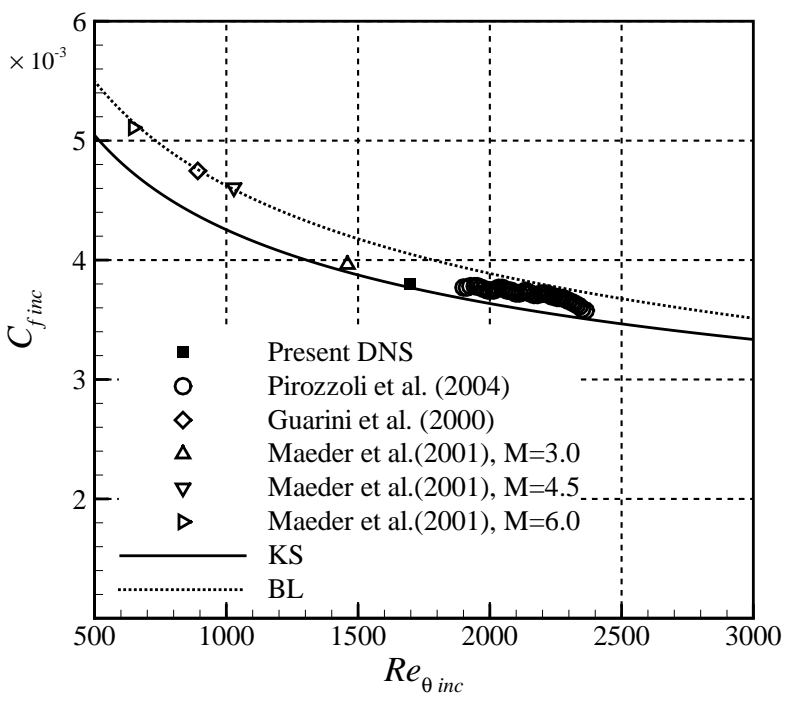

FiguRE 7. Distribution of friction coefficient as a function of Reynolds number based on momentum thickness, where 'KS' and 'BL' denote friction laws of Kámán-Schoenherr and Blasisus, respectively.

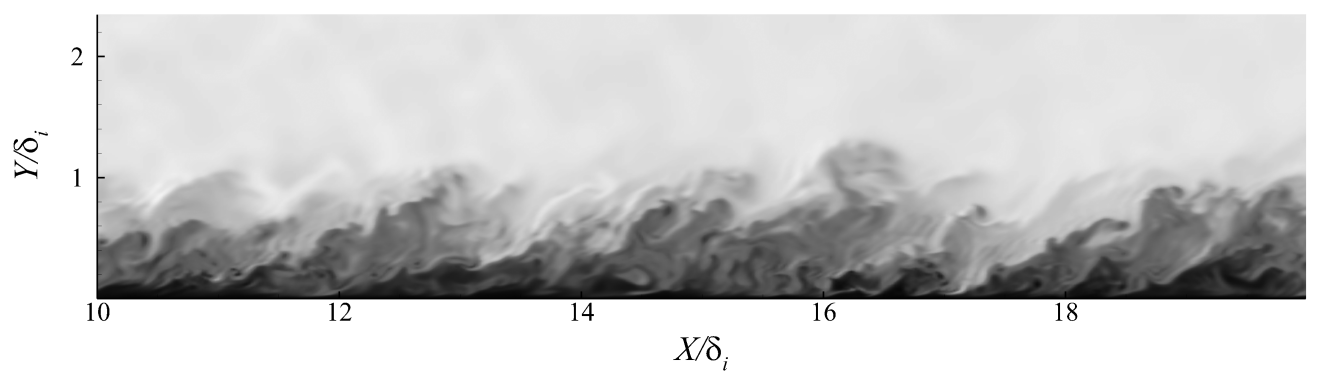

FigURE 8. Instantaneous distribution of density in the supersonic flat-plate boundary layer acquired from DNS

$$
\begin{gathered}
C_{f_{i n c, K S}}=\frac{1}{17.08\left(\log _{10} R e_{\theta_{i n c}}\right)^{2}+25.11 \log _{10} R e_{\theta_{i n c}}+6.012}, \\
C_{f_{i n c, B L}}=\frac{0.026}{R e_{\theta_{i n c}}^{1 / 4}}
\end{gathered}
$$

Obviously, the skin friction data obtained from present DNS replicate the correct skin friction trend with $R e_{\theta_{i n c}}$. This further confirms the accuracy of the present data and also the reliability of the van Driest II transformation in collapsing skin friction data at different Mach numbers.

Figure 8 gives an instantaneous distribution of density in the supersonic flat-plate boundary layer acquired from DNS. Fine structures within the turbulent boundary layer are clearly resolved, indicating that the DNS code and the simulation setup used in the present investigation are capable of revealing both the instantaneous and statistical information of a supersonic turbulent boundary layer.

To further validate the DNS data in the concave region, the streamwise velocity profiles 


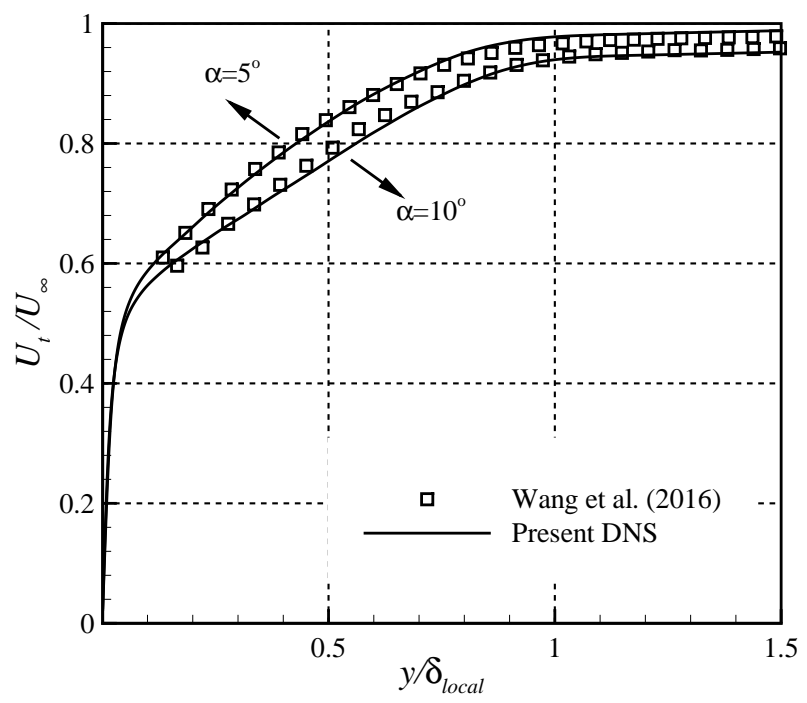

FIgURE 9. Comparisons of mean streamwise velocity profiles at two streamwise positions $\left(\alpha=5.0^{\circ}\right.$ and $\left.\alpha=10.0^{\circ}\right)$ in the concave region with that of Wang et al. $(2016 a)$. The streamwise velocity is normalized with the free stream velocity $U_{\infty}$ and the wall-normal height is normalized with the local boundary layer thickness $\delta_{\text {local }}$.

at two streamwise positions are compared with our previous experimental results (Wang et al. 2016a) acquired from Particle Image Velocimetry (PIV), as shown in figure 9. The wall-normal position is normalized by the local boundary layer thickness $\delta_{\text {local }}$. Regarding the definition and streamwise variation of $\delta_{\text {local }}$, they will be discussed in Section 3.1.1. It can be found in the figure that the computational results agree well with the experimental. The streamwise velocity is apparently reduced in the main stream.

\section{Results}

\subsection{Mean properties}

\subsubsection{Boundary layer thickness}

The contour map of averaged static pressure of the supersonic concave boundary layer is given in figure 10. Obviously, the static pressure varies in both of the streamwise and wall-normal directions due to the downstream leaning compression waves. The wall-normal variation of static pressure leads to the wall-normal variation of the free stream velocity, making it impossible to define the boundary layer thickness using the conventional $99 \%$ criterion, as shown in figure 11 where the outer edge defined by $99 \% U_{\infty}$ is highlighted with an orange line. By assuming a constant static pressure within the boundary layer, Fernholzh \& Finley (1980) introduced a fictitious boundary-layer edge state, through which the boundary layer thickness could be deduced. However, as we can find in figure 10, the static pressure varies within the boundary layer. Thus, the method introduced by Fernholzh \& Finley (1980) cannot be used here to define the boundary layer thickness. It is necessary to have a new and more physically based method to define the boundary layer thickness.

The method to define the boundary layer thickness described in the present investigation is deduced from the distribution of principal strain rate. The contour map of the normalized mean principal strain rate $S_{\text {prin }}=\partial\left(U_{t} / U_{\infty}\right) / \partial\left(y / \delta_{i}\right)$ is given in figure 11 . 


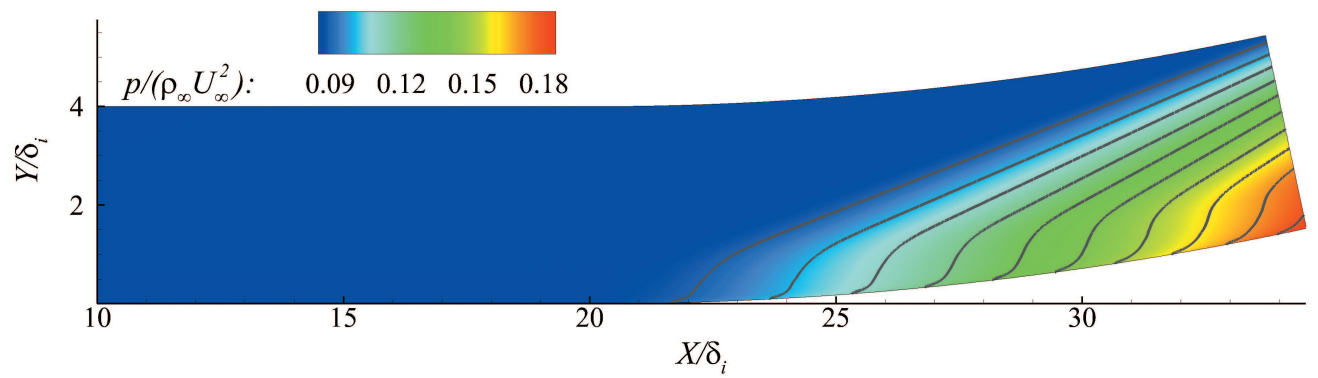

Figure 10. Contour map of the averaged static pressure. The pressure is normalized with the free stream parameter $\rho_{\infty} U_{\infty}^{2}$.

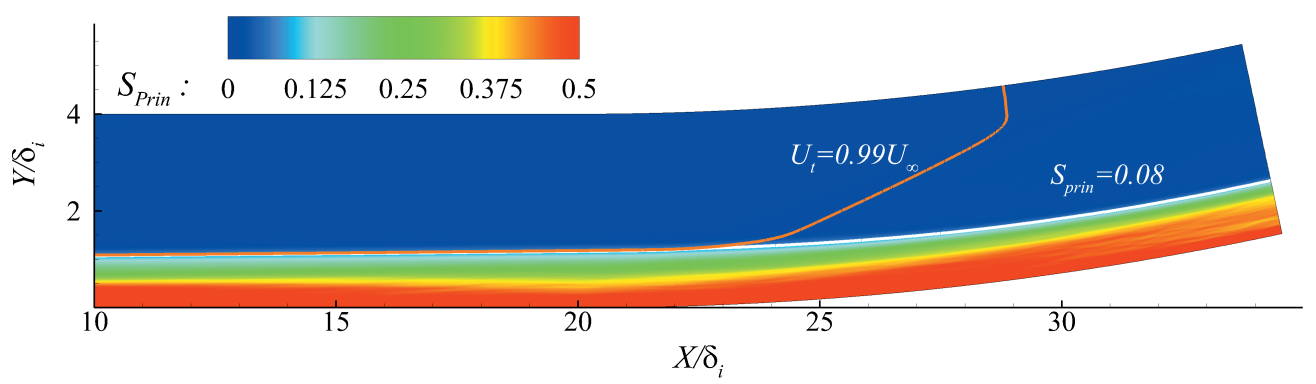

FiguRE 11. Contour map of the normalized principal strain rate $S_{\text {prin }}=\partial\left(U_{t} / U_{\infty}\right) / \partial\left(y / \delta_{i}\right)$.

Clearly, the strain rate within the boundary layer is much larger than that in the main flow. Within main flow, $S_{\text {prin }}$ is in the order of $10^{-4}$, while within the boundary layer, the $S_{\text {prin }}$ is much larger. Thus, it is possible to define the boundary layer thickness physically by choosing an appropriate threshold of the principal strain rate.

In figure 12(a), the streamwise variations of boundary layer thickness under five appropriate $S_{\text {prin }}$ thresholds, which are $S_{\text {prin }}=0.06,0.07,0.08,0.09$ and 0.10 are given. Obviously, with different thresholds, absolute values of the boundary layer thickness vary, but the streamwise varying trends are similar. The variation of the boundary layer thickness around the streamwise position of $x=2 \delta_{i}$ is due to relaxation of the turbulent inflow generated by the digital-filter-based method. Here, we choose the threshold of $S_{\text {prin }}=0.08$ to define the boundary layer thickness. The outer edges of the boundary layer defined by $S_{\text {prin }}=0.08$ and $S_{\text {prin }}=0.08$ are plotted in the figure 11 . As can be found in the figure, the edges defined by $99 \% U_{\infty}$ and $S_{\text {prin }}=0.08$ are close in the flat-plate region and in the near field downstream of the turning point. Further downstream of $X \approx 23 \delta_{i}$, while $S_{\text {prin }}=0.08$ still defines a physical edge, the edge defined by $99 \% U_{\infty}$ moves fast into the main stream. The streamwise variation of streamwise velocity component at the outer edge is given in figure 12(b). In the flat-plate region, the edge-velocity keeps a nearly constant value of $0.99 U_{\infty}$ in the streamwise direction and it agrees well with the conventional $99 \%$ criterion. In the concave region, the edge-velocity decreases due to the compression.

\subsubsection{Wall friction}

Streamwise variations of the skin friction coefficient $C_{f}=\tau_{w} /\left(0.5 \rho_{\infty} U_{\infty}^{2}\right)$ and the normalized friction velocity $U_{\tau}=u_{\tau} / U_{\infty}$ are given in figures $13(\mathrm{a})$ and $13(\mathrm{~b})$, respectively. The streamwise increase of wall friction before $x=7 \delta_{i}$ is due to the flow relaxation of the digital-filter-generated inflow. In the flat-plate region, a slow decrease of the wall friction 

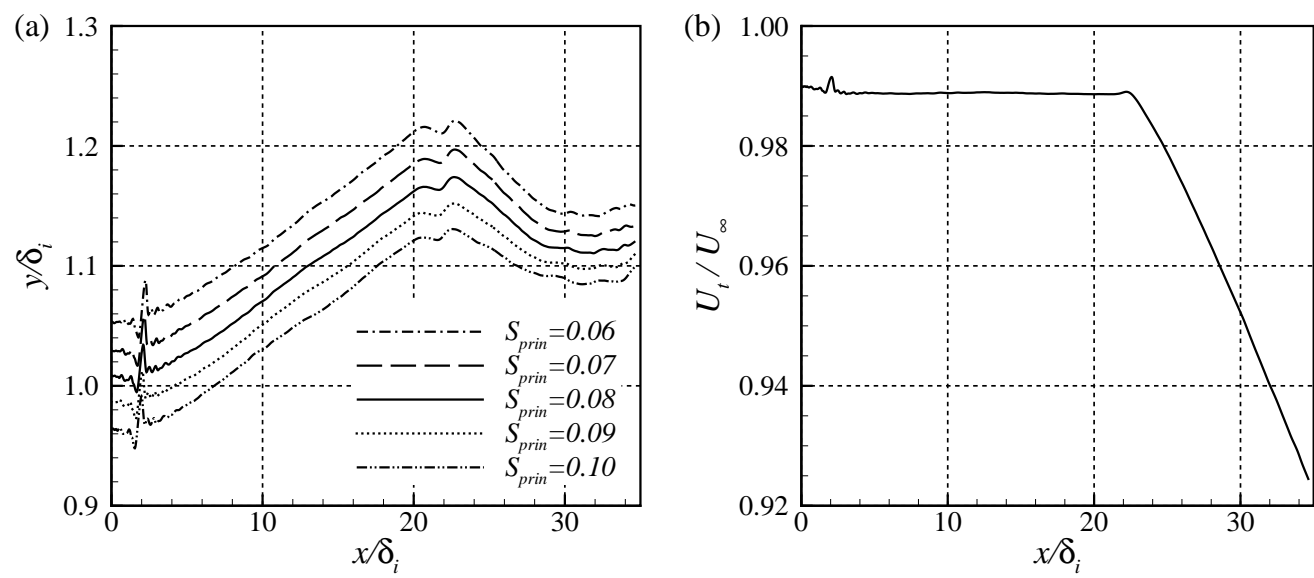

FiguRE 12. (a) Streamwise variations of boundary layer thickness with different thresholds of normalized principal strain rate; (b) streamwise variation of streamwise velocity component at the outer edge of the boundary layer, which is defined by the threshold of $S_{\text {prin }}=0.08$. The velocity is the normalized value by $U_{\infty}$

is found. In the concave region, it rises with the wall turning, which is consistent with the finding given by Donovan et al. (1994). However, if we carefully examine the curve, we could find that the wall friction actually undergoes a slight drop in the vicinity of the turning point before it rises. This is mainly caused by the additional drag imposed by the suddenly emerged concave wall at the turning point, which leads to the decrease of near-wall velocity and the related wall friction. We will find in subsequent discussion that the streamwise increase of skin friction in the concave region is mainly due to the promoted turbulence which transports the high-momentum flow in the outer layer to the near-wall region and thus increases the near-wall velocity gradient in the wall-normal direction. However, at the turning point the turbulence has not been effectively promoted to increase the near-wall velocity, which cannot compensate the velocity loss brought by the additional drag. As a result, the streamwise velocity at $x=20 \delta_{i}$ is finally reduced, as well as the skin friction. In the concave region, despite of the streamwise increase of wall shear, the friction velocity decreases with the wall turning. Considering that the friction velocity is calculated with the relation $u_{\tau}=\sqrt{\tau_{w} / \rho_{w}}$, the decrease of the $U_{\tau}$ is due to the increase of flow density.

\subsubsection{Velocity profiles}

Velocity profiles with inner and out scaling are shown in figures 14 and 15, respectively. Results are presented at five streamwise positions, $x=18.3 \delta_{i}, \alpha=3.0^{\circ}, 6.0^{\circ}, 9.0^{\circ}$ and $11.0^{\circ}$, where the streamwise position $x=18.3 \delta_{i}$ is in the flat-plate region (the concave turning point is at $x=20 \delta_{i}$ ) and $\alpha=3.0^{\circ}, 6.0^{\circ}, 9.0^{\circ}$ and $11.0^{\circ}$ denoted by the local wall turning angle in the concave region. Local friction velocity are used for the inner scaling, while local boundary layer thicknesses are used for the outer scaling.

In figure 14, we can find that at all streamwise positions the viscous sub-layer is well resolved and the profiles collapse for $y^{+} \lesssim 8$. Compared with the incompressible results of Barlow \& Johnston (1988b) where the profiles collapse well up to $y^{+} \approx 50$, the impact of the concave curvature is found to be able to get down to the region much closer to the wall under the supersonic condition. From the buffer layer and above, the influence of the concave wall on the velocity profile is visible. Though it is still possible to find the 

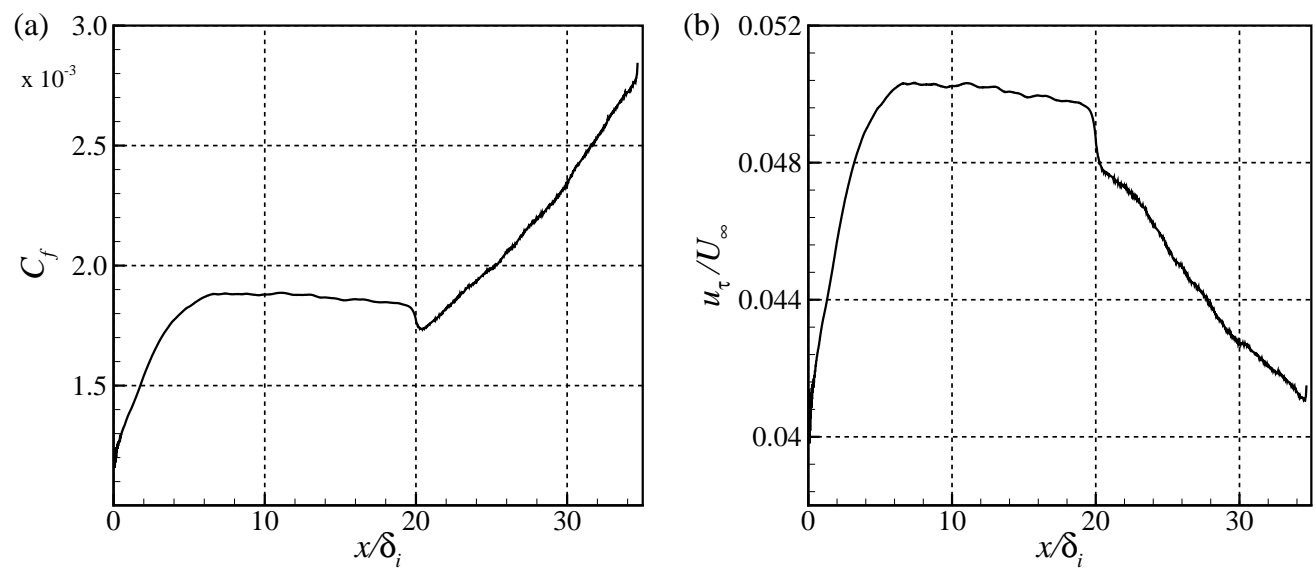

Figure 13. Streamwise variations of (a) friction coefficient $C_{f}=\tau_{w} /\left(0.5 \rho_{\infty} U_{\infty}^{2}\right)$ and (b) friction velocity normalized by $U_{\infty}$.

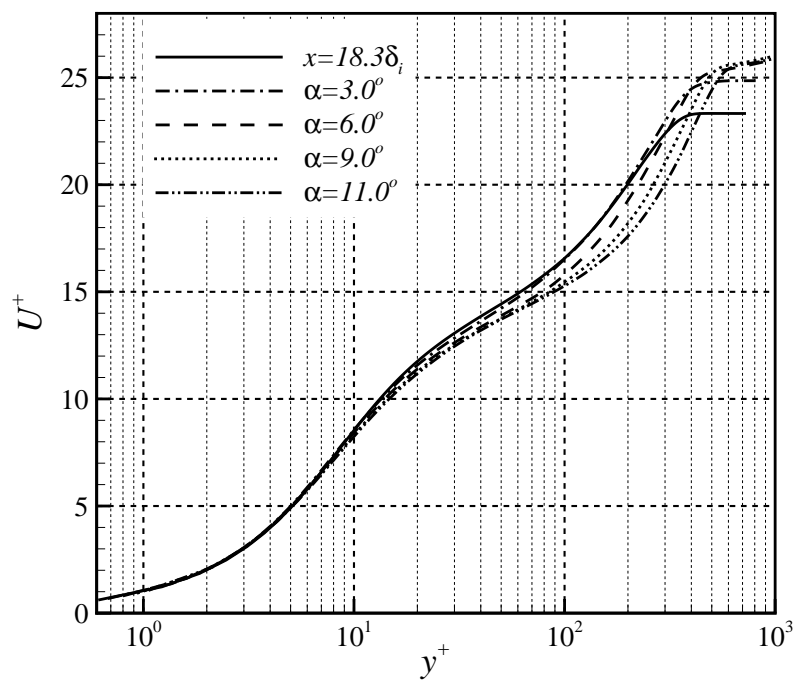

Figure 14. Profiles with inner scaling at different streamwise positions. Streamwise positions in the concave region are denoted by the local turning angle of wall.

logarithmic region with constant slope on the concave boundary layer, the slope decreases as flow develops on the concave surface.

In figure 15(a), a general view of velocity profiles throughout the boundary layer is presented. A zoom-in plot of profiles in the near-wall region is given in figure $15(\mathrm{~b})$. Due to the compression, the streamwise velocity decreases for $y / \delta_{\text {local }} \gtrsim 0.03$ as flow develops on the concave surface. However, in the near-wall region $\left(y / \delta_{\text {local }} \lesssim 0.03\right)$, the streamwise velocity increases with the wall turning, as shown in figure 15(b). This explains the streamwise increase of wall friction in the concave boundary layer. To facilitate subsequent analysis, we denote the region $y / \delta_{\text {local }} \lesssim 0.03$ as Layer $\mathrm{A}$, and the region $y / \delta_{\text {local }} \gtrsim 0.03$ as Layer B. Discussions on the physical cause of the alteration of the velocity profile in the concave region are given in Section 4. 

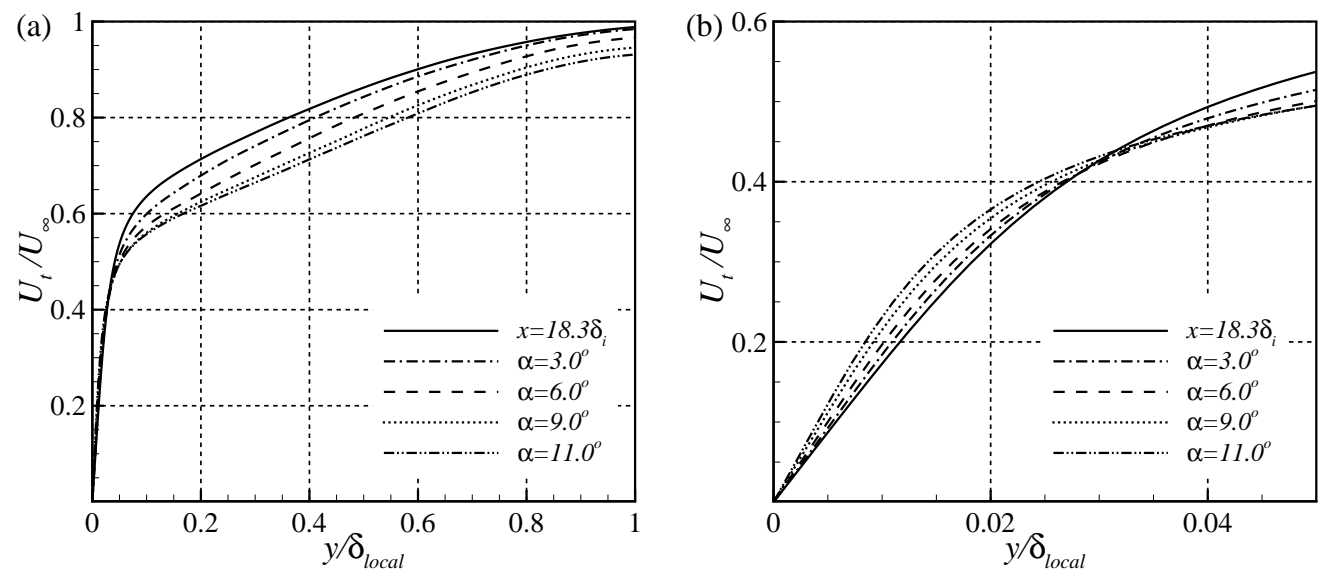

Figure 15. Profiles with outer scaling at different streamwise positions. The wall-normal positions are normalized by the local boundary layer thickness. (a) Velocity profiles within the height of $y / \delta_{\text {local }}<1.0$; (b) velocity profiles in the near-wall region within the height of $y / \delta_{\text {local }}<0.05$.

\subsubsection{Strain rate}

To have a clear understanding of the mean shear stress throughout the concave boundary layer, profiles of the normalized principal strain rate $S_{\text {prin }}$ at five streamwise locations are presented in figure 16. In figure 16(a), the wall-normal position is scaled with inner variables, while in figures 16(b) and 16(c), the wall-normal position is scaled with the local boundary layer thickness.

The Principal strain rate $S_{\text {prin }}$ increases with the wall turning in the near-wall region (see figure 16 (a) and (b)). The shear stress within the viscous sub-layer is much higher than that in the logarithmic and wake regions. Outside the viscous sub-layer, $S_{\text {prin }}$ drops quickly. Different from the sub-layer where the velocity gradient is maintained by the viscosity, the rapid growth of turbulent intensity outside the viscous sub-layer gradually makes the turbulent viscosity to be the dominant factor in turbulent transportation and dissipation. The observed drop of $S_{\text {prin }}$ with the increase of wall-normal position in the buffer layer is the result of the raised Reynolds shear stress which quickly smoothes the velocity gradient.

Considering that $S_{\text {prin }}$ has much higher value in the near-wall region, profiles of $S_{\text {prin }}$ are given within different ranges of height to have a clear presentation of the principal strain data. In figure $16(\mathrm{~b})$, the data are given in the near-wall region of $y / \delta_{\text {local }}<0.1$, while in figure $16(\mathrm{c})$, the data are given within $0.1 \leqslant y / \delta_{\text {local }} \leqslant 1.5$. Combining figures 16(b) and 16(c), it is clear that at different wall-normal positions, the streamwise varying trends of $S_{\text {prin }}$ are different as well. Three layers with different streamwise varying trend of $S_{\text {prin }}$ can be identified. This is consistent with the experimental finding given by Wang et al. (2016a). However, due to limit of spatial resolution of PIV, the high-shear region within the boundary layer is not revealed in their experiments and the layers cannot be clearly defined in their work. This can be clarified from the current numerical results, and the three layers are divided as follows.

(1) Layer I, located in the region of $y / \delta_{\text {local }} \lesssim 0.012$ and $S_{\text {prin }}$ grows with the wall turning;

(2) Layer II, located in the region of $0.015 \lesssim y / \delta_{\text {local }} \lesssim 0.2$ and $S_{\text {prin }}$ decreases with the wall turning; 
(3) Layer III, located in the region of $0.4 \lesssim y / \delta_{\text {local }} \lesssim 0.8$ and $S_{\text {prin }}$ increase with the wall turning.

By combining figures 15 and 16, a complete scenario of the streamwise varying trend of velocity as well as its variation with height can be constructed. The streamwise increase of $S_{\text {prin }}$ in Layer I is closely related to the near-wall streamwise growth of velocity in Layer A. But the thickness of Layer I is smaller than that of Layer A. This indicates that the streamwise growth rate of velocity varies with the wall-normal position in Layer A. In Layer I, the higher the wall-normal position is, the faster the velocity grows in the streamwise direction, offering a streamwise increasing trend of $S_{\text {prin }}$. While in the region higher than the Layer I, the velocity growth gradually slows down with the increase of wall-normal height and its streamwise varying trend is entirely reversed when it gets into the region higher than $y / \delta_{\text {local }} \approx 0.03$ (the lower edge of Layer B). As a consequence, a streamwise decreasing trend of $S_{\text {prin }}$ in Layer II is observed. Further higher to the position of $y / \delta_{\text {local }} \approx 0.2 \sim 0.4$, although the streamwise decreasing trend of velocity is maintained, it slows down gradually. Thus a higher wall-normal position can give a lower reduction rate of velocity, leading to the noted streamwise increasing trend of $S_{\text {prin }}$ in Layer III.

An averaged contour map of the normalized bulk dilatation $(\nabla \cdot \mathbf{U}) \cdot \delta_{i} / U_{\infty}$ is given in figure 17 . The trace of the first compression wave induced by the concave wall are clearly extracted in the contour map. It seems that the bulk dilatation is very sensitive in extracting simple waves. The compression wave is curved within the boundary layer and in the main flow it extends straightly towards downstream. The negative value noted in the region downstream of the first compressive wave indicates that the flow is compressed and the flow farther away from the wall is subjected to more severe compression.

\subsection{Turbulent statistics}

Profiles of r.m.s. of the streamwise velocity component scaled by $1 / U_{\infty}$ are plotted against $y / \delta_{\text {local }}$ at five streamwise positions in figure $18(\mathrm{a})$, where $(u u)_{r m s}=\sqrt{\overline{u^{\prime 2}}} / U_{\infty}$. In figure 18(b), profiles of the scaled r.m.s. $(u u)_{r m s}^{+}$are plotted versus $y^{+}$. The profiles of r.m.s. of the wall-normal and the spanwise velocity components are shown similarly in figures 19 and 20, respectively. Similarly, the profile of $\overline{u^{\prime} v^{\prime}}$ scaled by $1 / U_{\infty}^{2}$ is plotted in figure 21 .

The absolute value of r.m.s. of the streamwise velocity component is found to increase with the wall turning in regions of $y / \delta_{\text {local }}<0.02$ and $0.2<y / \delta_{\text {local }}<1.0$, as shown in figure 18(a). An emerging secondary bump of $(u u)_{r m s}$ can be observed in the outer layer. The near-wall peak value, which located in the region of $0.02<y / \delta_{\text {local }}<0.1$, changes in a more complicated way. While from the flat-plate region to the streamwise position of $\alpha=3.0^{\circ}$ the peak value is increased, it is reduced further downstream in the concave region. With inner scaling, the concave curvature promotes $(u u)_{r m s}^{+}$throughout the boundary layer, as shown in figure 18(b). In the concave region, profiles at different streamwise positions collapse for $y^{+}<10$, within which the mean velocity profiles are also noted to collapse. A secondary peak of $(u u)_{r m s}^{+}$is found to gradually emerge around the wall-normal position of $y^{+} \approx 200$ (corresponding to $y / \delta_{\text {local }} \approx 0.3$ ).

Profiles of r.m.s. of the wall-normal velocity are given in figure 19. Clearly, $(v v)_{r m s}$ is promoted by the concave curvature throughout the boundary layer, as shown in figure 19(a). The peak position moves slightly outward with the wall turning, which is also the case under inner inner scaling, as given in figure 19(b). Similar to $(u u)_{r m s}^{+},(v v)_{r m s}^{+}$ at different streamwise positions in the concave region is also found to collapse within $y^{+} \lesssim 30$. 

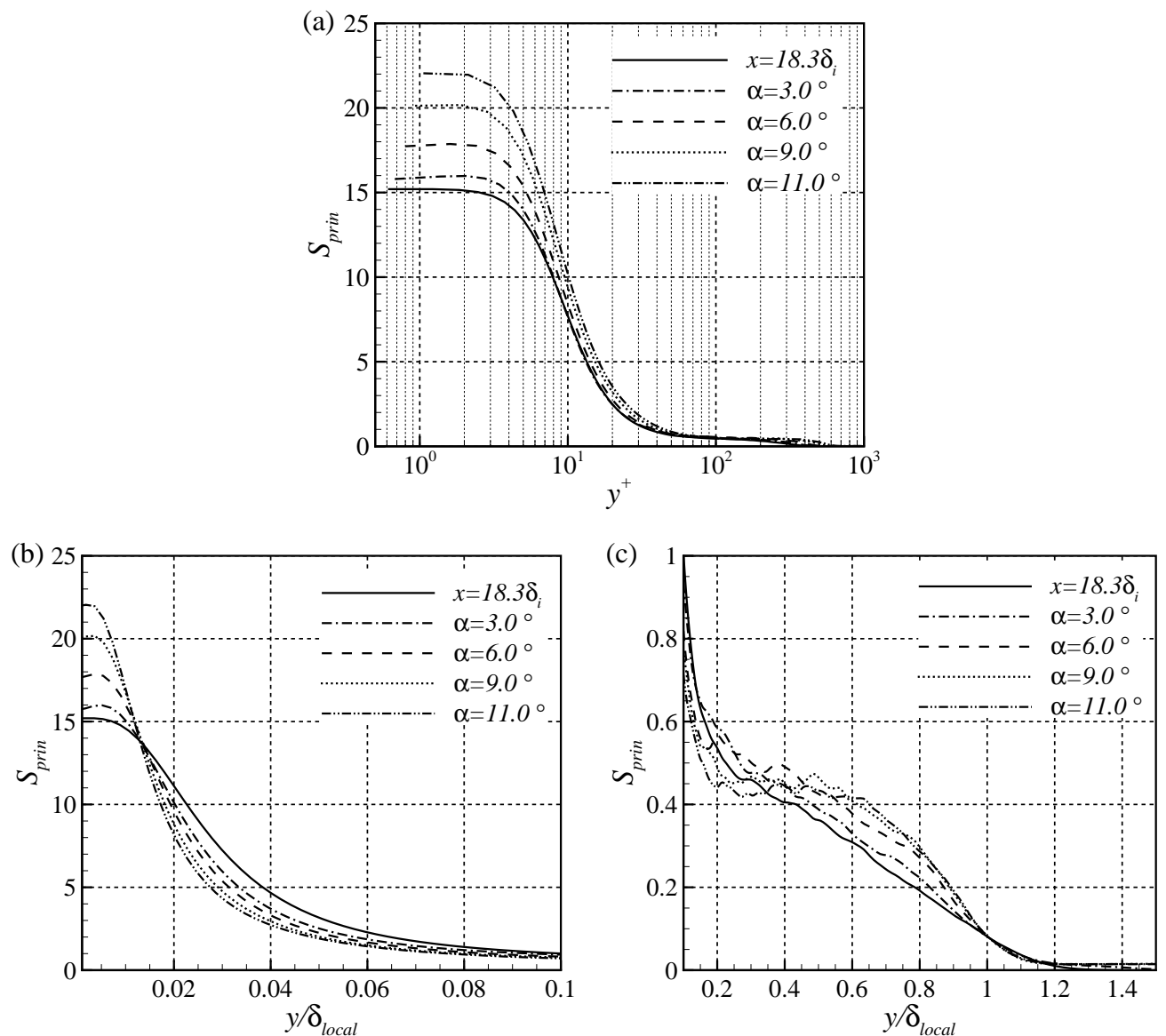

Figure 16. Profiles of the normalized principal strain rate at different streamwise positions. The wall-normal coordinates are normalized with different ways. (a) The wall-normal positions are denoted by $y^{+}$; (b) data is given in the near-wall region within the height of $y / \delta_{\text {local }}<0.1$; (c) data is given within the height of $0.1 \leqslant y / \delta_{\text {local }} \leqslant 1.5$.

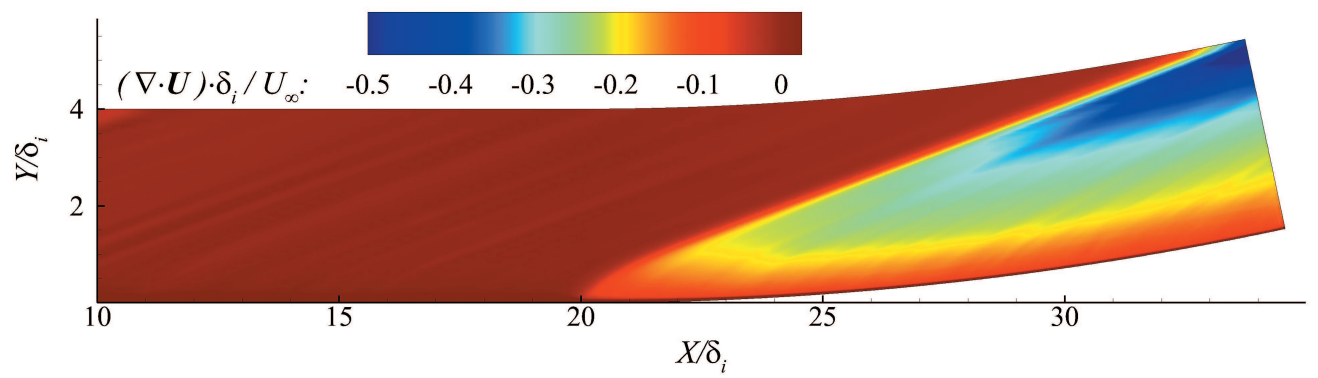

Figure 17. Contour map of the normalized bulk dilatation $(\nabla \cdot \mathbf{U}) \cdot \delta_{i} / U_{\infty}$.

Profiles of spanwise turbulent intensity are given in figure 20. With different ways of normalization, the spanwise turbulent intensity is found to increase in the streamwise direction throughout the boundary layer. Profiles of $(w w)_{r m s}^{+}$do not collapse in the nearwall region. Similar to that of the streamwise turbulent intensity, a secondary bump of spanwise turbulent intensity also found to gradually emerge around $y^{+} \approx 200$. 
18

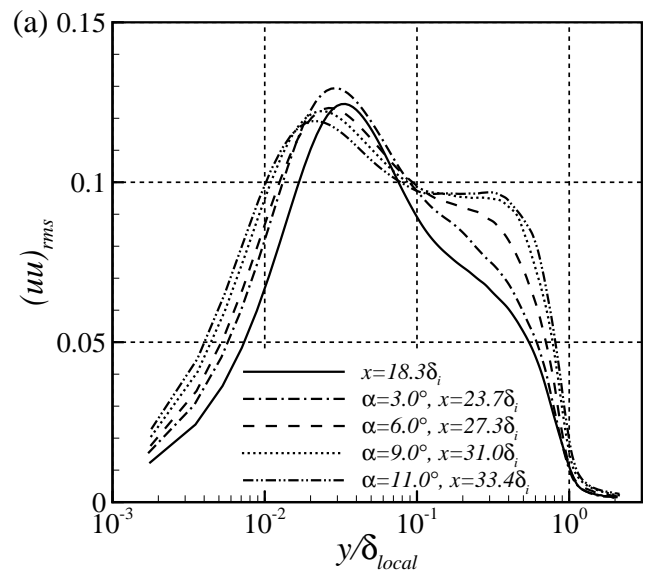

Q. C. Wang and others

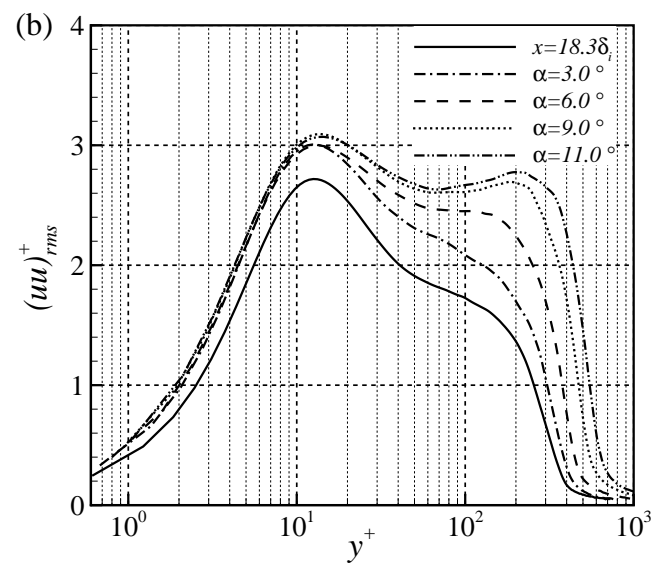

FiguRE 18. Profiles of r.m.s. of the streamwise velocity component at five streamwise positions. Profiles are presented with different ways of normalization: (a) the r.m.s. is scaled by $1 / U_{\infty}$ and given as $(u u)_{r m s}=\sqrt{\overline{{u^{\prime 2}}^{2}}} / U_{\infty}$, and the wall-normal coordinate is given as $y / \delta_{\text {local }}$; (b) the r.m.s. is given as $(u u)_{r m s}^{+}$and the wall-normal coordinate is given as $y^{+}$.

(a)

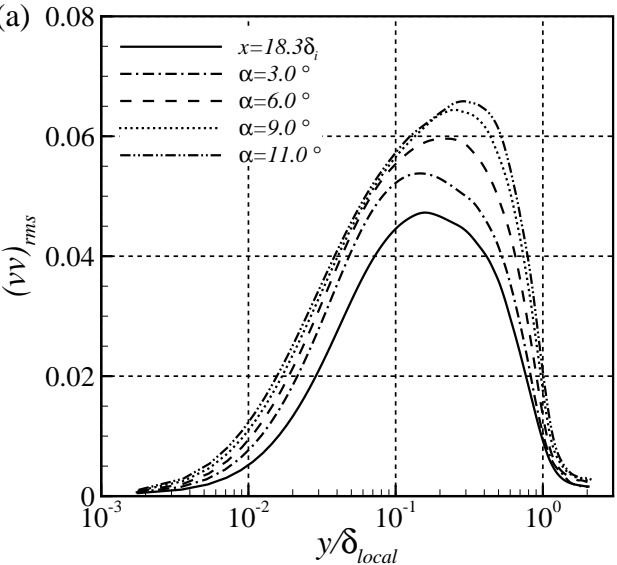

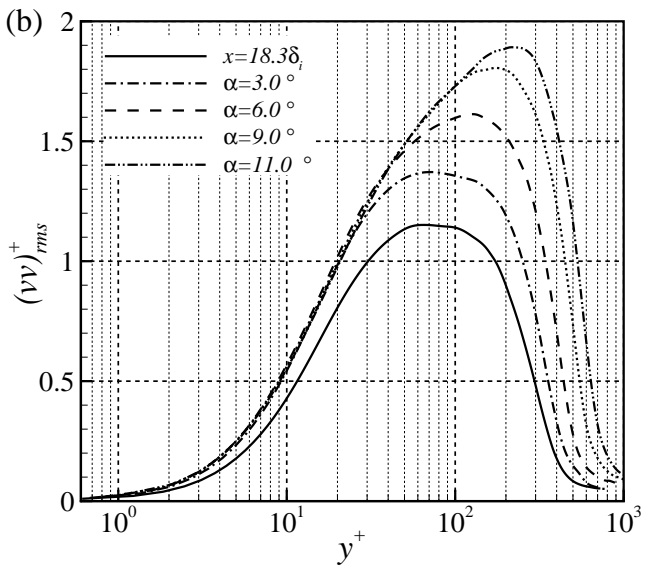

Figure 19. Profiles of r.m.s. of the wall-normal velocity component at five streamwise positions. Profiles are presented with different way of normalization: (a) streamwise variation of $(v v)_{r m s}=\sqrt{\overline{v^{\prime 2}}} / U_{\infty}$ with $y / \delta_{\text {local }} ;(\mathrm{b})$ streamwise variation of $(v v)_{r m s}^{+}$with $y^{+}$.

Profiles of scaled $\overline{u^{\prime} v^{\prime}}$ are given in figure 21. With outer scaling, the streamwise variation of $(u v)$ (see figure 21(a)) looks similar to that of $(u v)^{+}$(see figure 21(b)). Lower than the hight of $y / \delta_{\text {local }} \approx 0.02$ and higher than the hight of $y / \delta_{\text {local }} \approx 0.2,(u v)$ grows with the wall turning. Within $0.02 \lesssim y / \delta_{\text {local }} \lesssim 0.1,(u v)$ is increased from the streamwise position of $x=18.3 \delta_{i}$ in the flat region to $\alpha=3.0^{\circ}$, while further downstream in the concave region it is reduced with the wall turning. With inner scaling, the profiles of $(u v)^{+}$ collapse lower than $y^{+} \approx 10$ in the curved region, as shown in figure $21(\mathrm{~b})$. In a fully developed turbulent boundary layer, there always exists a region with nearly constant $(u v)^{+}$, which corresponds to the logarithmic region, as can be found in the profile at $x=18.3 \delta_{i}$. But in the concave turbulent boundary layer, the region with constant $(u v)^{+}$ does not exist anymore. $(u v)^{+}$are significantly increased by the concave curvature and the peak region also gradually moves outwards to $y^{+} \approx 200$. 

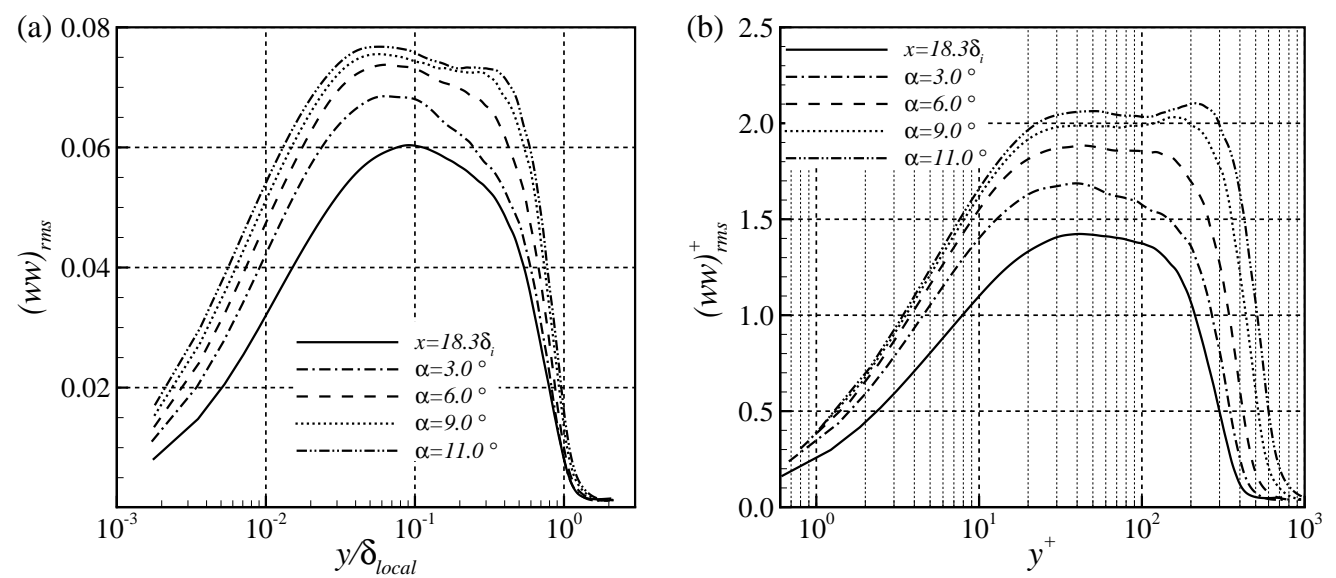

Figure 20. Profiles of r.m.s. of the spanwise velocity component at five streamwise positions. Profiles are presented with different way of normalization: (a) streamwise variation of $(w w)_{r m s}=\sqrt{\overline{w^{\prime 2}}} / U_{\infty}$ with $y / \delta_{\text {local }}$; (b) streamwise variation of $(w w)_{r m s}^{+}$with $y^{+}$.
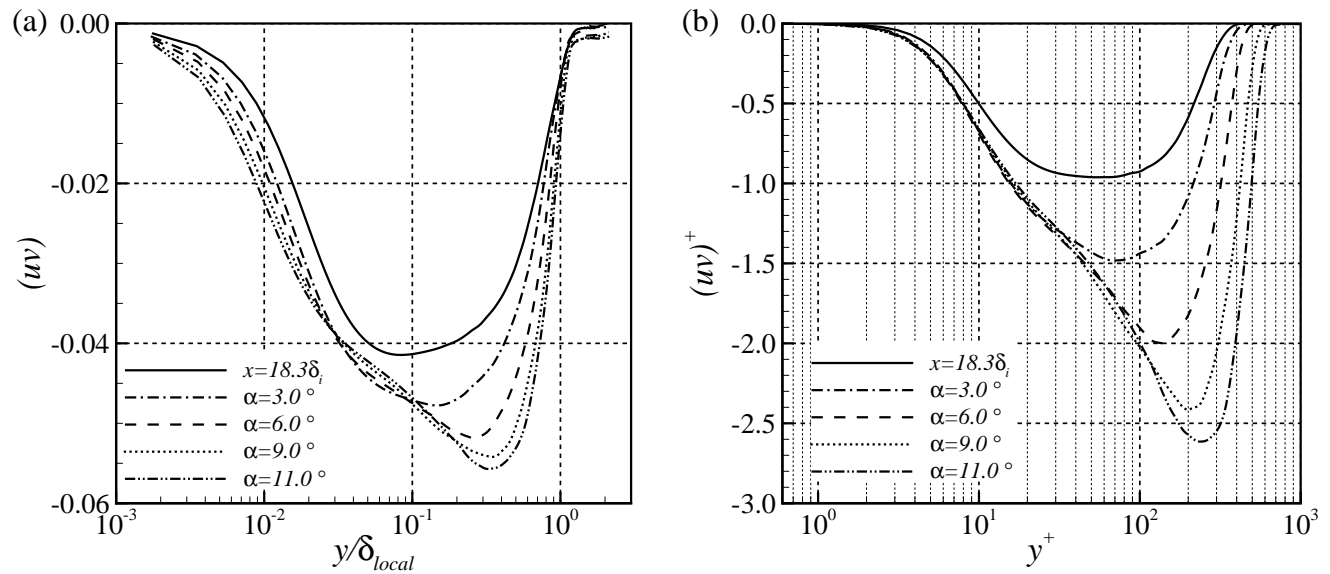

Figure 21. Profiles of scaled $\overline{u^{\prime} v^{\prime}}$ at five streamwise positions: (a) scaled by $1 / U_{\infty}^{2}$ and profiles of $(u v)=\overline{u^{\prime} v^{\prime}} / U_{\infty}^{2}$ versus $y / \delta_{\text {local }}$; (b) profiles of $(u v)^{+}$versus $y^{+}$.

Through above analysis of the turbulent statistics, we find that the secondary bumps in the profiles of $(u u)_{r m s}^{+}$and $(w w)_{r m s}^{+}$, and the peak positions of $(v v)_{r m s}^{+}$and $(u v)^{+}$ are all located at similar wall-normal positions around $y^{+} \approx 200 \sim 300$ in the wake region, which is interesting. Regarding the turbulent kinetic energy production for a low Reynolds number zero-pressure-gradient boundary layer, it is usually recognized that the peak production occurs within the buffer layer, at a wall-normal distance $y^{+} \approx 12$ (Smits et al. 2011), which is responsible for the inner bumps. According to the results given by Marusic et al. (2010), the $(u u)_{r m s}^{+}$profile can be considered to be the sum of two modes: a small viscous-scaled component primarily located in the near wall region, and a larger outer-scaled component peaking in the outer layer. In a high Reynolds number boundary layer, the turbulent intensity can be significantly promoted in the outer layer through the larger outer-scaled contribution (Marusic et al. 2010; Hutchins et al. 2009). In previous studies on the boundary layer subjected to the streamwise adverse pressure gradient, Monty et al. (2011) and Harun et al. (2013) also found the emergence of secondary peak 


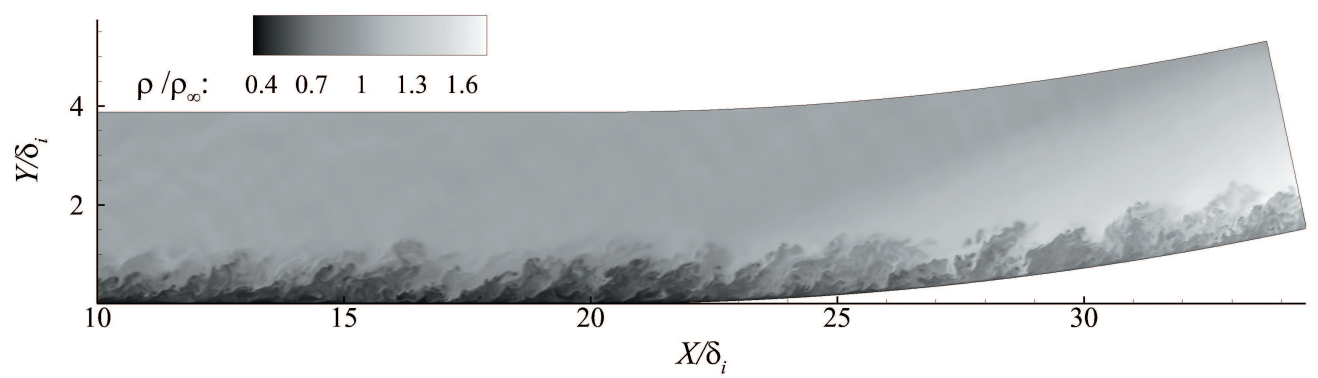

FigURE 22. Instantaneous distribution of density in the supersonic concave turbulent boundary layer

in the turbulent intensity profiles. Through checking the contributions of small and large scale components, they also attribute the secondary bump to the energised large-scale motions in the outer layer, which is similar to the high Reynolds number boundary layer. The strengthening of large-scale motions should be the same cause of the secondary bumps observed in present study. However, regarding how the large-scale motions are strengthened remains unclear. We will try to reveal this in Section 3.3.1.

The collapse of profiles of $(u u)_{r m s}^{+},(v v)_{r m s}^{+}$and $(u v)_{r m s}^{+}$at different streamwise positions for $y^{+}<10$ in the concave region suggests a new near-wall equilibrium state of turbulent production in the concave boundary layer. By carefully examining profiles of the turbulent statistics, it can be found that although they collapse in the concave region, they are all evidently higher than those in the flat-plate region. This hints that the new near-wall equilibrium state of the concave boundary layer is different from that of the flat-plate boundary layer. For $(w w)_{r m s}^{+}$, the profiles at different streamwise positions in the concave region do not collapse in the concave region, but it can be found that the differences between adjacent profiles are decreasing with the wall turning. Actually, the profiles at $\alpha=9^{\circ}$ and $\alpha=11^{\circ}$ have been very close in the near-wall region. This suggests that while the streamwise and wall-normal turbulent fluctuations have fast response to the concave curvature and reach the new equilibrium state quickly, the near-wall spanwise fluctuation is seemed to take much longer distance to reach a new equilibrium state.

\subsection{Turbulent structure}

\subsubsection{Instantaneous flow structures}

An instantaneous distribution of density in an XY plane of the supersonic concave turbulent boundary layer is given in figure 22. A streamwise increase of density brought by the compressive waves can be clearly seen. The streamwise variation of turbulent structures along the concave wall is similar to the flow-visualization results given by Wang \& Wang (2016). In the concave region, the vortex structures appear to have smaller scales than those in the flat-plate region. Considering that the shock waves could complicate the flow, without shock waves being introduced in present study, it can facilitate the direct study of physical impacts of the streamwise concave curvature and the related adverse pressure gradient on boundary layer.

An overall view of the instantaneous Euler vortices extracted with $\Delta$ criterion (Chakraborty et al. 2005; Chong et al. 1990), which clearly shows the three-dimensional evolution of the vortex structures under concave curvature, is given in figure 23(a). For $d / \delta_{i} \leqslant 0.4$, zoom-in views of the extracted vortices in the flat-plate and curved regions are given in figure 23(b) and figure 23(c), respectively. The difference of vortices in the flat-plate and curved regions are notable. Since the results are presented in the region 
(a)
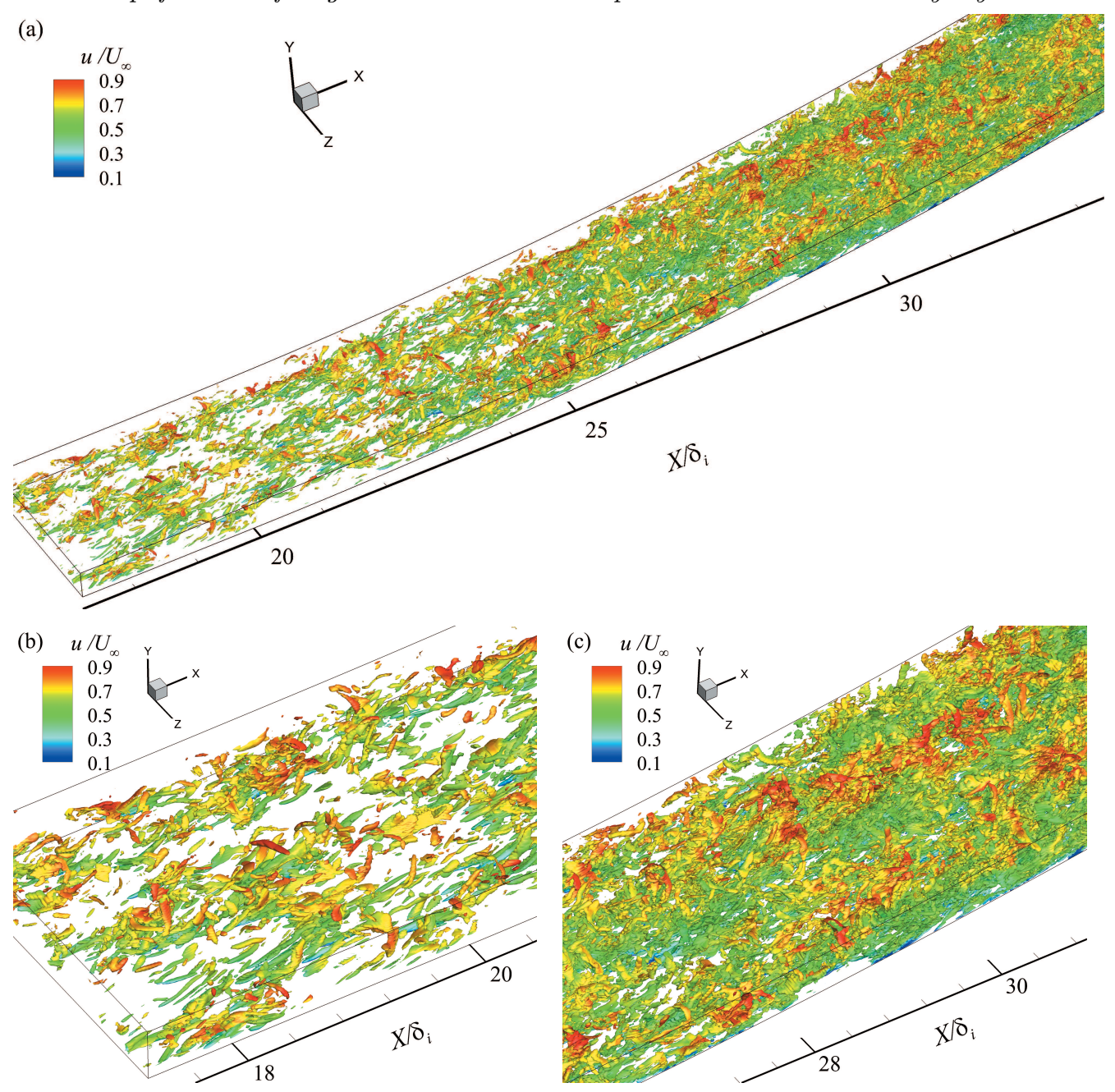

FIGURE 23. Instantaneous vortex structures extracted by $\Delta$ criterion with $\Delta=0.0015$ lower than the wall-normal height of $d / \delta_{i}=0.4$. The iso-surfaces are colored by the streamwise velocity. (a) an overall view of the vortex structures from the flat-plate region to the concave, (b) an zoomed view in the flat-plate region, (c) an zoomed view in the concave region.

relatively close to the wall, quasi streamwise vortices, whose existence is a typical feature of the near-wall turbulence, can be frequently noticed in the flat-plate region (see figure 23(b)). However, in the concave region (see figure 23(c)), turbulence is dominated by the well-developed hairpin vortices, which means that coherent large-scale motions are getting closer to the wall due to the concave curvature. The curvature gives higher vortex production, as a consequence much denser distributed vortices in the curved region are noticed.

In figure 24, cross-flow slices are shown for the instantaneous distribution of streamwise velocity at six streamwise positions. Two slices are given in the flat-plate region and four in the curved region. It is clear that the general distribution of the streamwise velocity in the concave region, especially at streamwise positions of $\alpha=9.0^{\circ}$ and $\alpha=11.0^{\circ}$, differs significantly from that in the flat-plate region. The low-momentum fluid which initially accumulated in the near-wall region is found to have more evident tendency to move 


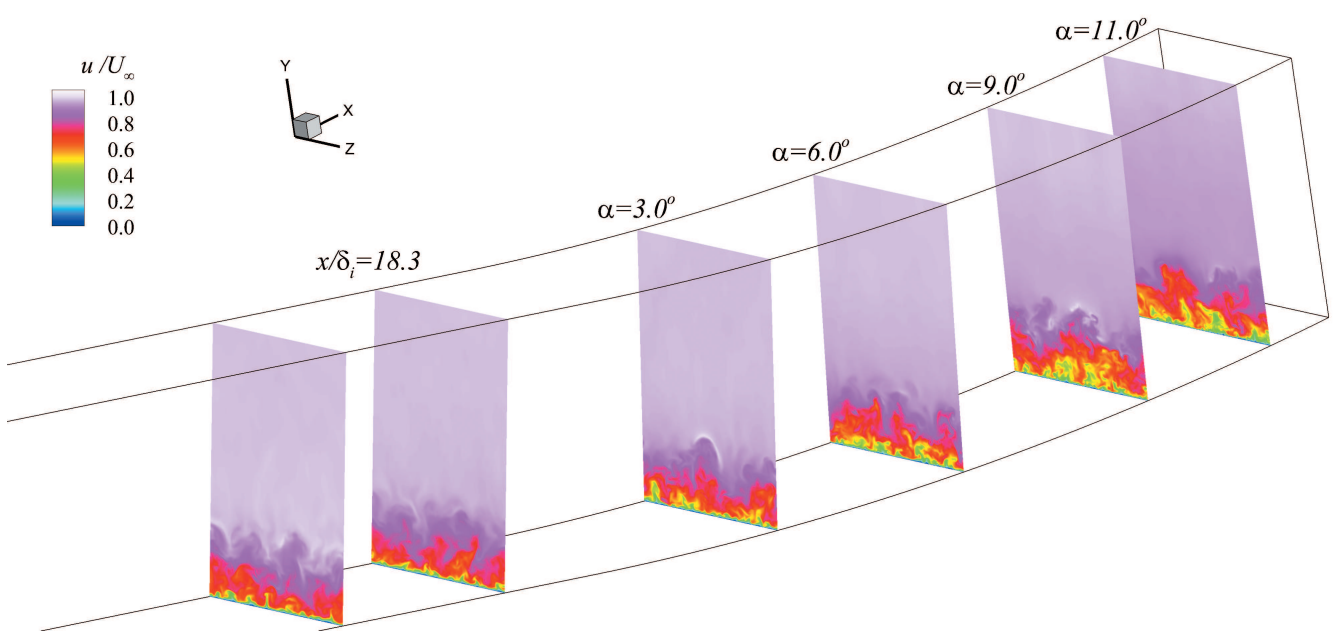

FIGURE 24. Instantaneous contour maps of streamwise velocity at different streamwise positions.

outwards under the impact of the concave curvature. The momentum transportation is seemed to be enhanced in the concave region. To have a more detailed discussion on the instantaneous flow field, two cross-sectional slices presented in figure 24 are further extracted, as shown in figure 25. One slice is in the flat-plate region at $x / \delta_{i}=18.3$ and the other is in the concave region at $\alpha=11.0^{\circ}$. Contour maps of the instantaneous streamwise velocity as well as streamtraces are presented in the figure. Bulges due to the outwards moving trend of the low-momentum flow can be seen in both of the flatplate and the concave regions, and it bulges further into the main stream in the concave region than that in the flat-plate region. Along with the bulge of the low-momentum flow, large-scale streamwise swirls can be clearly seen through the pattern of the streamtraces at $\alpha=11.0^{\circ}$ (see figure 25(b)). While in the flat-plate region, the streamwise swirls are seemed to be more randomly distributed and in smaller scale(see figure 25(a)). The obvious differences in velocity distribution and streamtrace pattern between the slices extracted at $x / \delta_{i}=18.3$ and at $\alpha=11.0^{\circ}$ suggest the existence of large-scale longitudinal roll cells which are first found by Barlow \& Johnston $(1988 a, b)$ in the incompressible concave boundary layer. According to Barlow \& Johnston $(1988 a, b)$, it is hard to define the roll cells in the turbulent boundary layer due to the complexity of flow. The roll cells do not have concentrated vorticity and they usually vary with time and streamwise position. More supportive evidences of the large-scale roll cells can be found by extracting instantaneous streamwise vortices and probability density distributions of streamwise vorticity, as given below.

For vortex extraction, a modified swirling-strength parameter $\lambda_{s, x}$ introduced by $\mathrm{Wu}$ \& Christensen (2006) is used. $\lambda_{s, x}$ is defined as

$$
\lambda_{s, x}=\lambda_{c i}(y, z) \frac{\omega_{x}(y, z)}{\left|\omega_{x}(y, z)\right|}
$$

where, $\omega_{x}=0.5(\partial w / \partial y-\partial v / \partial z) \cdot \delta_{i} / U_{\infty}$ is the dimensionless streamwise vorticity and $\lambda_{c i}$ is the imaginary part of the complex eigenvalue of the local velocity-gradient tensor (Zhou et al. 1999; Elsinga et al. 2010). The sign of the vorticity is used to distinguish the direction of rotation. In the cross-sectional plane and for the streamwise vortices, 
(a)

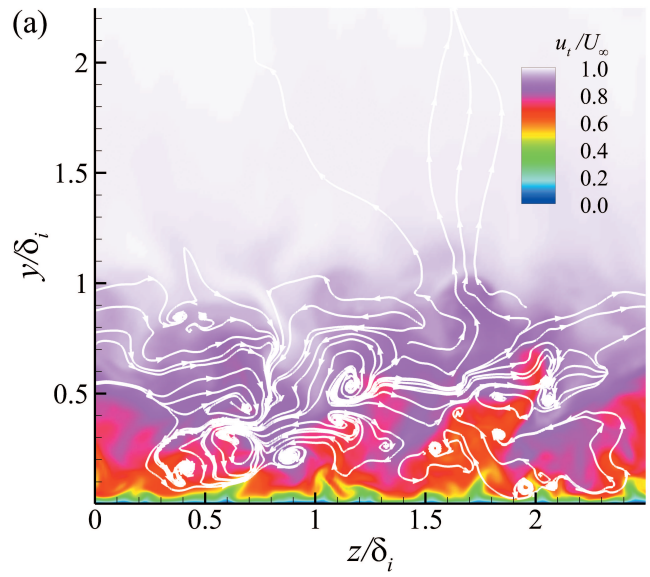

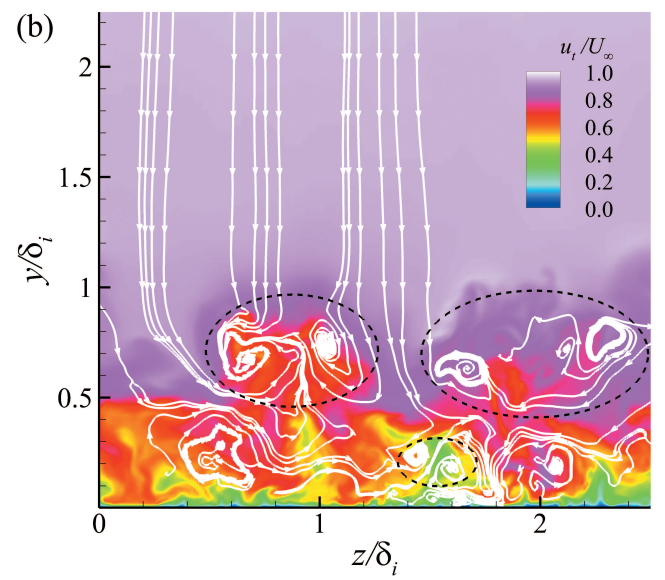

Figure 25. Contours of the instantaneous streamwise velocity and streamtraces in two cross-sectional planes: (a) $x / \delta_{i}=18.3$, (b) $\alpha=11.0^{\circ}$.

the streamwise swirling strength $\lambda_{c i}(y, z)$ considers only the spanwise and wall-normal velocity components and is defined as the imaginary part of the eigenvalues of $J_{v w}$

$$
J_{v w}=\left[\begin{array}{cc}
\frac{\partial v}{\partial y} & \frac{\partial v}{\partial z} \\
\frac{\partial w}{\partial y} & \frac{\partial w}{\partial z}
\end{array}\right]
$$

Contour maps of the instantaneous swirling-strength parameter $\lambda_{s, x}$ corresponding to the velocity distributions presented in figure 25 are given in figure 26 . As can be found in the figure, throughout the boundary layer the number and strength of the streamwise vortices in the concave region are higher than that in the flat-plate region. The streamwise swirling is intensified by the concave curvature. The more densely distributed streamwise vortices observed here is consistent with the results given in figure 23 where much larger number of hairpins are observed in the concave region. Combining the streamtraces presented in figure 25 and streamwise vortices in figure 26, it is reasonable to deduce that it is actually the accumulations of a large number of small-scale streamwise vortices with similar sense of rotation and their combined effects that leads to the formation of the large-scale roll cells.

In figure 27, distributions of probability density of the dimensionless streamwise vorticity $\omega_{x}$ at two wall-normal positions $\left(y / \delta_{i}=0.008\right.$ and $\left.y / \delta_{i}=0.23\right)$ are presented. The results show promotion of the streamwise swirling in the concave boundary layer. At both wall-normal positions the boundary layer in the concave region tends to have larger streamwise vorticity. More dominant promotion of streamwise vorticity is found in the near-wall position. This statistical result supports the deduction from figure 25 . It is because of the combined effect of these streamwise vortices which are intensified in the concave boundary layer that forms the large-scale roll cells. Through intensifying the pre-existed streamwise vortices and producing more, the overall streamwise swirling is promoted. Typical features of the roll cells are the clear spanwise variations of turbulent structure. The bulges of the low-speed flow are the directed results of the promoted streamwise swirling which gives the low-speed flow stronger tendency to move outwards and induces the high-speed flow to move towards the wall.

By checking the directions of swirling in the plane of $\alpha=11.0^{\circ}$, it can be observed that streamtraces on each side of one low-momentum bulge actually swirl in counter 
(a)

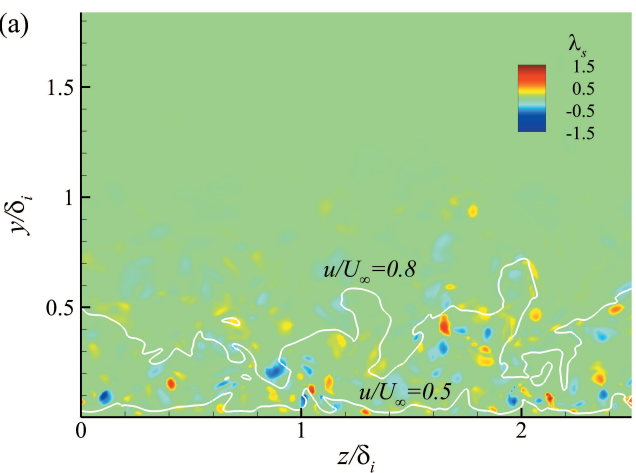

Q. C. Wang and others

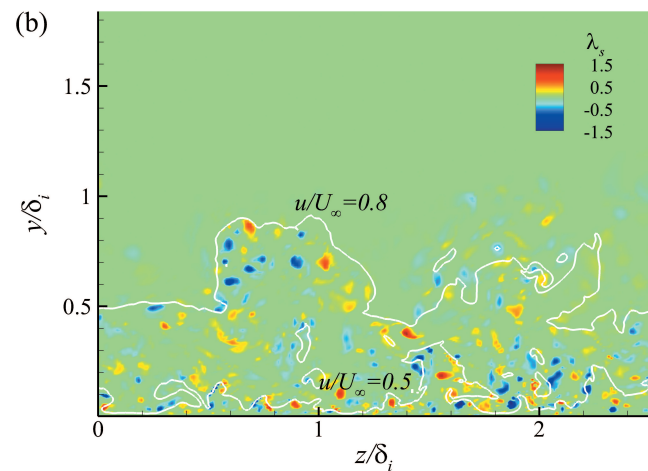

Figure 26. Contours of instantaneous swirling-strength parameter $\lambda_{s, x}$ at two streamwise positions: (a) $x / \delta_{i}=18.3$, (b) $\alpha=11.0^{\circ}$. Contour lines of $u / U_{\infty}=0.5$ and 0.8 are also plotted.

(a)

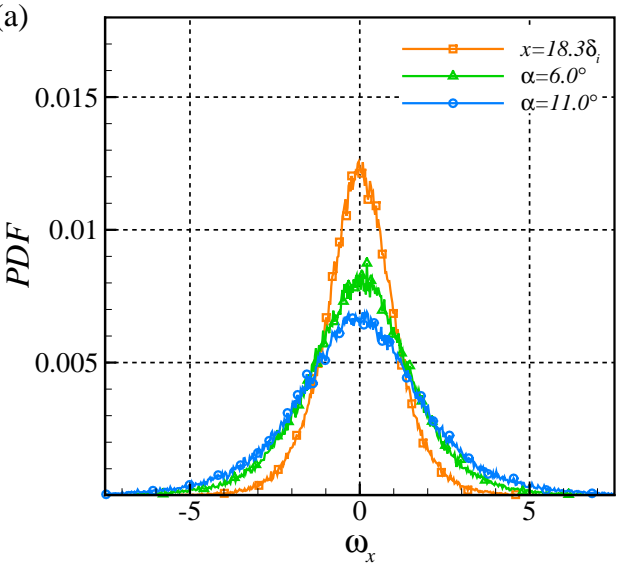

(b)

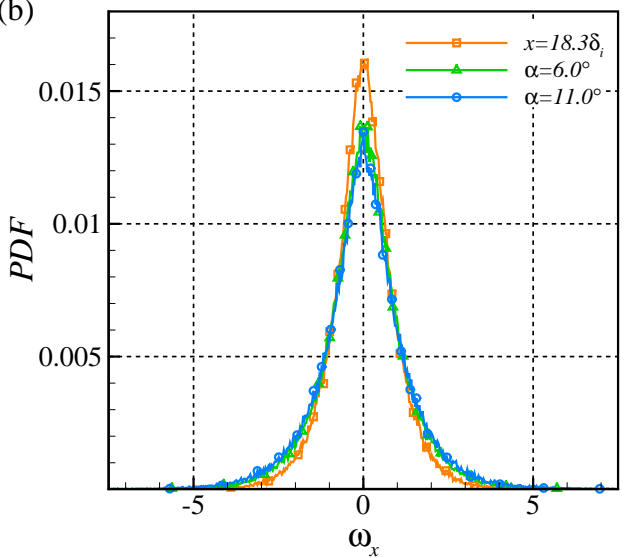

FiguRe 27. Probability density distributions of dimensionless streamwise vorticity $\omega_{x}$ at two wall-normal position: (a) $y / \delta_{i}=0.008$ in the viscous sub-layer, (b) $y / \delta_{i}=0.23$ around the outer edge of the $\log$ region. Symbols are used to discriminate the curves acquired at different streamwise positions.

directions, as shown in the circled region on figure 25(b). This resembles the flow field with Görtler vortices. It has been generally recognized that the Görtler instability introduced by the streamline concave curvature has significant impact on both turbulent and laminar boundary layers. In the laminar boundary layer the Görtler instability presents itself as the counter-rotating streamwise vortices, while in the turbulent boundary layer it has similar impacts on the flow by promoting the streamwise swirling. Though it is impossible to find the Görtler vortices in the turbulent boundary layer, the intensification of streamwise vortices found here indicates the contribution of the Görtler instability to the formation of large-scale roll cells in the supersonic flow. This is similar to that found that by Barlow \& Johnston $(1988 a, b)$ in the incompressible concave boundary layer.

To find out more details about how these large-scale roll cells are formed, an instantaneous iso-surface of the streamwise velocity at $u / U_{\infty}=0.55$ is obtained, as shown in figure 28. The iso-surface is colored by the wall-normal height. Dominant stronger outwards moving trend of the low-momentum flow in the concave region than that in the flat-plate region can be observed in the figure, indicating the significant role of the large-scale roll cells in promoting momentum transportation. It can also be found in 


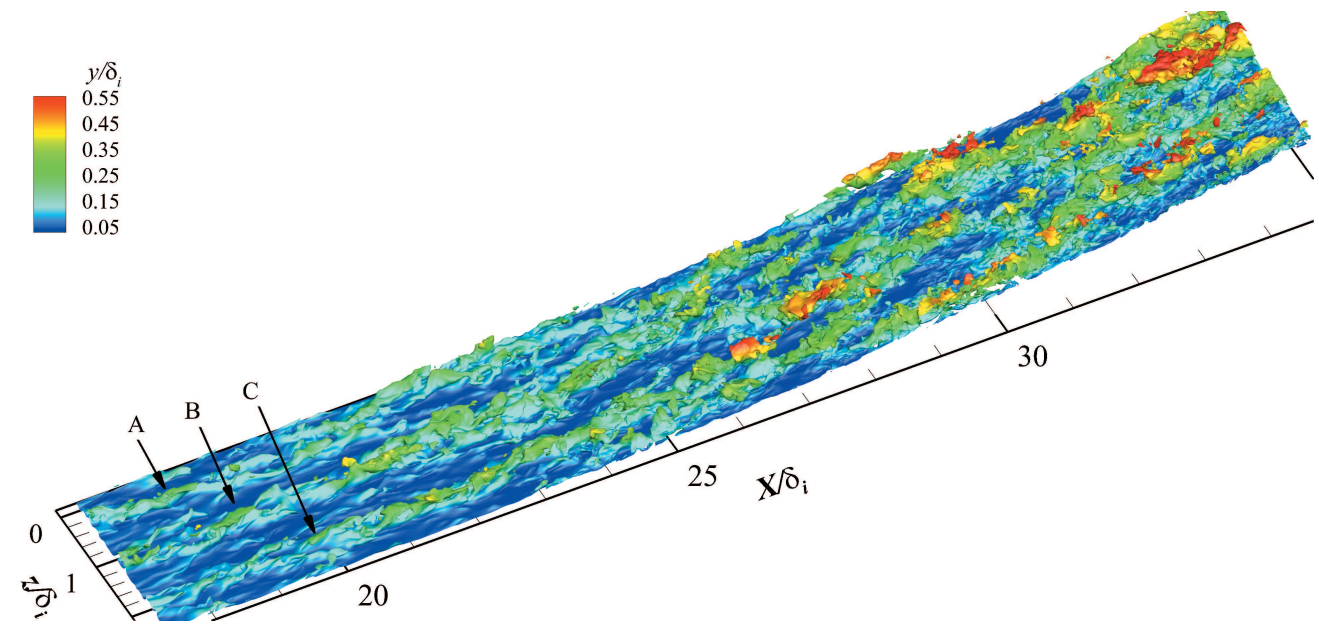

FIGURE 28. Iso-surface of streamwise velocity at $u / U_{\infty}=0.55$ colored with the wall-normal position.

figure 28 that the the bulges of iso-surface are actually evolved from the streamwise extended structures (denoted by A, B and C) formed in the flat-plate boundary layer, which remind us of the very-large-scale motions(VLSMs) and their possible relationship with the large-scale roll cells found in the concave boundary layer.

Another view of these streamwise extended structures, which is acquired at $y / \delta_{i}=0.12$ in the logarithmic region (the corresponding $y^{+}$is 38 in the flat region), is given in figure 29 (a). Vortices extracted with $\Delta=0.0015$ and contours of the streamwise velocity are presented in the figure. Similar to that shown in figure 28, three elongated low-momentum regions which start in the flat-plate region and persist for the entire streamwise length of the viewing window can be clearly observed. Despite meandering of the low-speed structures in the concave region, the extracted vortices are seen to distribute mainly along these low-speed structures, suggesting the dominant contribution of the low-speed structures in the generation of vortices. A zoomed-in view of the flow field within $15 \leqslant$ $x / \delta_{i} \leqslant 25$ is given in figure $29(\mathrm{~b})$, where contour lines of the streamwise velocity at $u_{t} / U_{\infty}=0.6$ are extracted. The streamwise swirling strength parameter $\lambda_{s, y}$ in the $x z$ plane is also calculated by using the relation similar to the equation 3.1 , and patches with $\left|\lambda_{s, y}\right| \geqslant 0.2$ are presented in the figure. It can be found in figure $29(\mathrm{~b})$ that the extracted vortices are mostly distributed along the streamwise extended low-momentum structures. For any of the three low-speed structures, negative $\lambda_{s, y}$ can be frequently observed around the upper edges while positive $\lambda_{s, y}$ is usually found around the lower edges. The counter-rotating of these vortices on two sides of the elongated low-speed region indicates the streamwise aligned one- and two-legged hairpin vortices and the related packets(Adrian et al. 2000; Elsinga et al. 2010). This also indicates that the streamwise extended low-speed structures in the logarithmic layer are the VLSMs.

As have been revealed in many previous reports regarding both supersonic and subsonic turbulent boundary layers (Ringuette et al. 2008; Kim \& Adrian 1999; Lee \& Sung 2011; Hutchins \& Marusic 2007), the VLSMs are brought by the combination of adjacent hairpin packets. The momentum transportation within the turbulent boundary layer is closely related to the induced velocity from the swirling of the hairpins which are organized in packets. While the high-momentum flow in the outer layer tend to be induced to move towards the wall, the near-wall low-momentum flow is induced to move away from the wall. The streamwise elongated regions of momentum deficit are the direct 
(a)
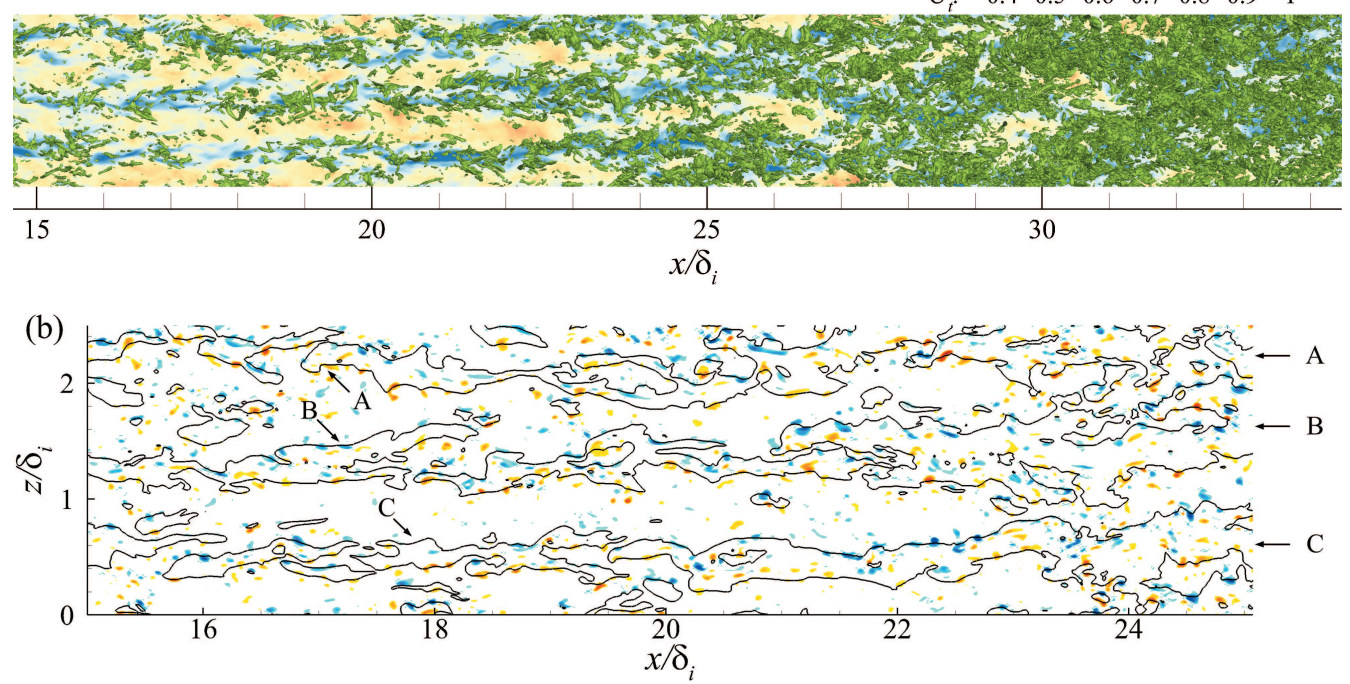

Figure 29. Contours of instantaneous field at wall-normal heights of $y / \delta_{i}=0.12$ (the corresponding $y^{+}$are 38 at the streamwise position of $x=18.3 \delta_{i}$ and 50 at $\alpha=6.0^{\circ}$ ). (a) Contours of streamwise velocity and vortex structures extracted with $\Delta=0.0015$. (b) Contour lines of streamwise velocity with $u_{t} / U_{\infty}=0.6$ and patches of swirling strength parameter $\left|\lambda_{s, y}\right| \geqslant 0.2$; The blue and red contours represent the positive and negative values of $\left|\lambda_{s, y}\right|$, respectively.

results of the streamwise aligned hairpin packets (Adrian et al. 2000). The bulges of isosurface of the streamwise velocity observed in figure 28 are brought by the out-moving low-momentum flow. In the concave region, the low-momentum flow bulge much higher, suggesting that the large-scale motion and the momentum transportation are intensified through the formation of roll cells in the concave boundary layer. Despite the stronger out-moving trend, these bulges remain streamwise extensions of the VLSMs formed in the flat-plate boundary layer.

Thus, it can be confirmed that the large-scale roll cells found in the concave boundary layer are actually originated from the VLSMs formed in the flat-plate boundary layer. Hairpin vortices which are usually organized in packets populate the VLSMs. The legs of the hairpin vortices are usually streamwise extended vortices. We have shown that the streamwise vortices can be significantly intensified in the concave boundary layer. After the VLSMs formed in the flat-boundary layer get into the concave region, the large number of the streamwise extended vortices within the hairpin packets are strengthened by the Görtler instability. And more hairpin vortices are generated around the edges of the low-speed regions. On two sides of the low-speed region, the hairpin vortices tend to have counter sense of rotation. After intensification of these vortices and more are generated by the Görtler instability, their accumulations and combined effect lead to larger-scale streamwise swirls, as that found in figure 25(b) and the roll cells observed by Barlow \& Johnston $(1988 a, b)$. The roll cells can significantly enhance the momentum transportation between the inner and outer layers and make the velocity iso-surface in the concave boundary layer bulges higher than that in the flat-plate boundary layer. Stronger momentum transportation makes stronger shear between the low- and high-momentum region. This also contributes to the generation of hairpin vortices in the low-speed region in the concave boundary layer, as can be seen in figures 23 and 29(a). 
(a)

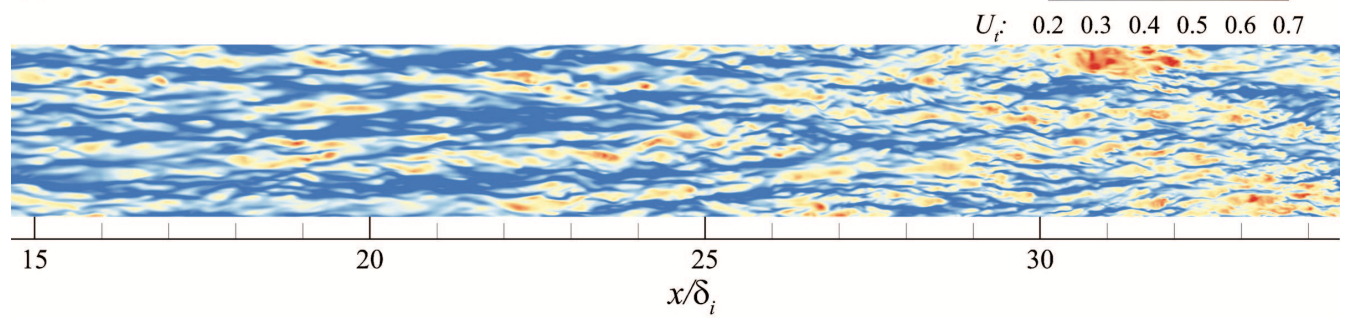

(b)

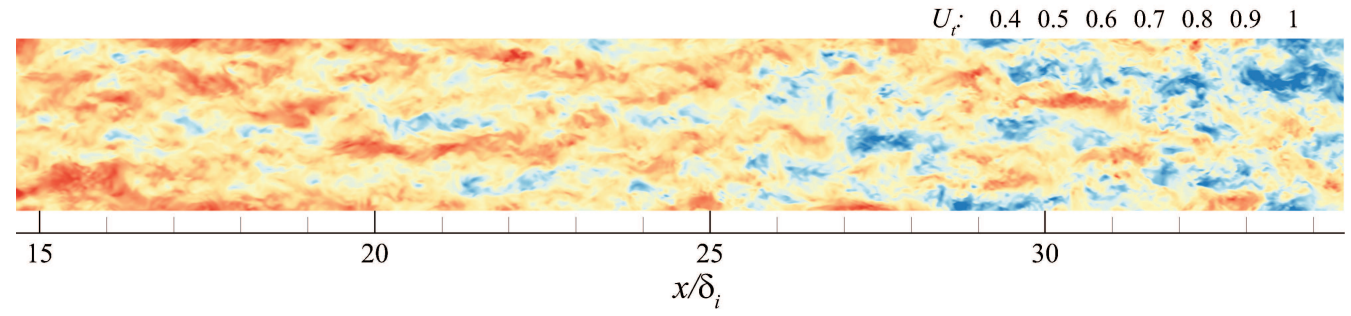

FigURE 30 . Contours of the streamwise velocity in wall-parallel planes at the wall-normal heights of (a) $y / \delta_{i}=0.02$ (the corresponding $y^{+}$are 6.5 at the streamwise positions of $x=18.3 \delta_{i}$ in the flat plate region and 7.5 at $\alpha=6.0^{\circ}$ in the concave region), and (b) $y / \delta_{i}=0.33$ (the corresponding $y^{+}$are 100 at the streamwise position of $x=18.3 \delta_{i}$ and 130.24 at $\left.\alpha=6.0^{\circ}\right)$.

The impacts of the roll cells on the near-wall turbulence can be seen in figure 30(a), where an contour map of instantaneous velocity at the wall-normal hight of $y / \delta_{i}=0.02$ (the corresponding $y^{+}$is 6.5 in the flat region) in the sub-layer is given. Due to the largescale roll cells, the near-wall structures of the turbulent boundary layer are dramatically altered. The streamwise elongated low-speed streaks can be clearly seen in the flat-plate region. In the concave region, the streaks are severely interrupted and truncated by the frequent occurred high-speed patches, making the scales of these streaks smaller in both of the streamwise and spanwise directions. The frequently noted high-speed patches in the curved region imply that while the outward motion is enhanced in the concave boundary layer, the inward motion is significantly promoted as well, through which the high-momentum flow is brought to the near-wall region. Though the turbulent structures in figures 30(a) and 29(a) are evidently different, the faint superimposed footprints of the VLSM and the large-scale roll cells in the near-wall region could be observed if we carefully examine their flow structures.

At the higher wall-normal position, as shown in figure 30(b) where an instantaneous distribution of streamwise velocity at $y / \delta_{i}=0.33$ (the corresponding $y^{+}$are 100 in the flat region) is given, the impact of the roll cells is also obvious. In the curved region, the low-momentum flow is found to have higher tendency to move outwards. While the turbulence is dominated by the high-momentum flow in the flat-plate region, downstream of $x / \delta_{i}=25$ the turbulence at the same wall-normal height is dominated by the flow with much lower velocity.

The formation of large-scale roll cells is the main cause of the promotion of turbulent level. Regarding how the turbulent level is promoted in the concave turbulent boundary layer is discussed in Section 3.3.2 


\subsubsection{Quadrant decomposition}

Due to the induced velocity from the swirl of the hairpin vortices which are organized in packets within the VLSMs and the large-scale roll cells, different turbulent events are induced. The quadrant decomposition technique ( $\mathrm{Lu} \&$ Willmarth 1973; Pokrajac et al. 2007; Tichenor et al. 2013) which has been widely used to study the dynamic boundary layer events is useful for the present investigation. With quadrant decomposition, the fluctuation data can be divided into four quadrants in the $x y$ plane: outward interaction (Q1 event, $u^{\prime}>0, v^{\prime}>0$ ); ejection (Q2 event, $u^{\prime}<0, v^{\prime}>0$ ); inward interaction (Q3 event, $u^{\prime}<0, v^{\prime}<0$ ); and sweep (Q4 event, $u^{\prime}>0, v^{\prime}<0$ ), where $u^{\prime}$ and $v^{\prime}$ are the streamwise and wall-normal velocity fluctuations.

Quadrant decomposition results at two wall-normal positions which are $y / \delta_{i}=0.008$ in the sub-layer and $y / \delta_{i}=0.04$ in the buffer layer are presented in figure 31. At each wall-normal position, scatter plots of the decomposition at three streamwise positions which are $x=18.3 \delta_{i}, \alpha=3.0^{\circ}$ and $\alpha=9.0^{\circ}$ are given.

Clearly, at $y / \delta_{i}=0.008$, the scattered area grows with the wall turning, as shown in figure 31 (a-c). The probability to have positive or negative $v^{\prime}$ with absolute value larger than $1.0 \% U_{\infty}$ is significantly increased as flow develops on the concave surface. For the streamwise fluctuation $u^{\prime}$, the probability to have a positive value larger than $15.0 \% U_{\infty}$ is increased with the wall turning. But the absolute value of negative $u^{\prime}$ is mostly confined to be smaller than $10.0 \% U_{\infty}$. The negative streamwise fluctuation are not significantly enhanced.

At the higher wall-normal position of $y / \delta_{i}=0.04$, as shown in figure 31 (d-f), the scattered areas are much larger than those at $y / \delta_{i}=0.008$. It should be noted that the vertical axis ranges for $v^{\prime} / U_{\infty}$ in figure 31 (d-f) are different from those in (a-c). The scattered area is also found to be increased with the wall turning, which is similar to that presented at $y / \delta_{i}=0.008$. The probability to have high positive or negative value of $u^{\prime}$ is not significantly increased. The streamwise increase of the scattered area is mostly attributed to the variation in the distribution of $v^{\prime}$ whose probability to have absolute value larger than $5.0 \% U_{\infty}$ is evidently increased in the concave region.

Through the analysis of figure 31, it is noticed that the wall-normal fluctuation at both heights is significantly promoted in the concave boundary layer. Combining this with the findings in Section 3.3.1, it is concluded that while the large-scale roll cells have significant contribution to the promotion of the wall-normal motion (both inward and outward motions), its impact on the streamwise motion is relatively weak. The ejection and sweep events are strengthened due to the promotion of wall-normal motion, which causes more intense momentum transfer between the inner and outer layers in the concave boundary layer.

The scatter plots also suggest that the Q2 and Q4 events take more important parts in the Reynolds stress. Following Tichenor et al. (2013), to further evaluate the respective contributions of different events, the average of $u^{\prime} v^{\prime}$ for each quadrant can be calculated.

$$
\overline{\left(u^{\prime} v^{\prime}\right)_{i}}=\frac{1}{N} \sum_{n=1}^{N_{i}}\left[\left(u^{\prime} v^{\prime}\right)_{i}\right]_{n}, i=1 \ldots 4,
$$

where, $N$ is the number of total samples, $N_{i}$ is the sample number for the $i$ th quadrant. The sum of averages of $u^{\prime} v^{\prime}$ for four quadrants is the Reynolds shear stress.

$$
\sum_{n=1}^{4} \overline{\left(u^{\prime} v^{\prime}\right)_{i}}=(u v)_{a v e}
$$


Amplification of large-scale motion in a supersonic concave boundary layer
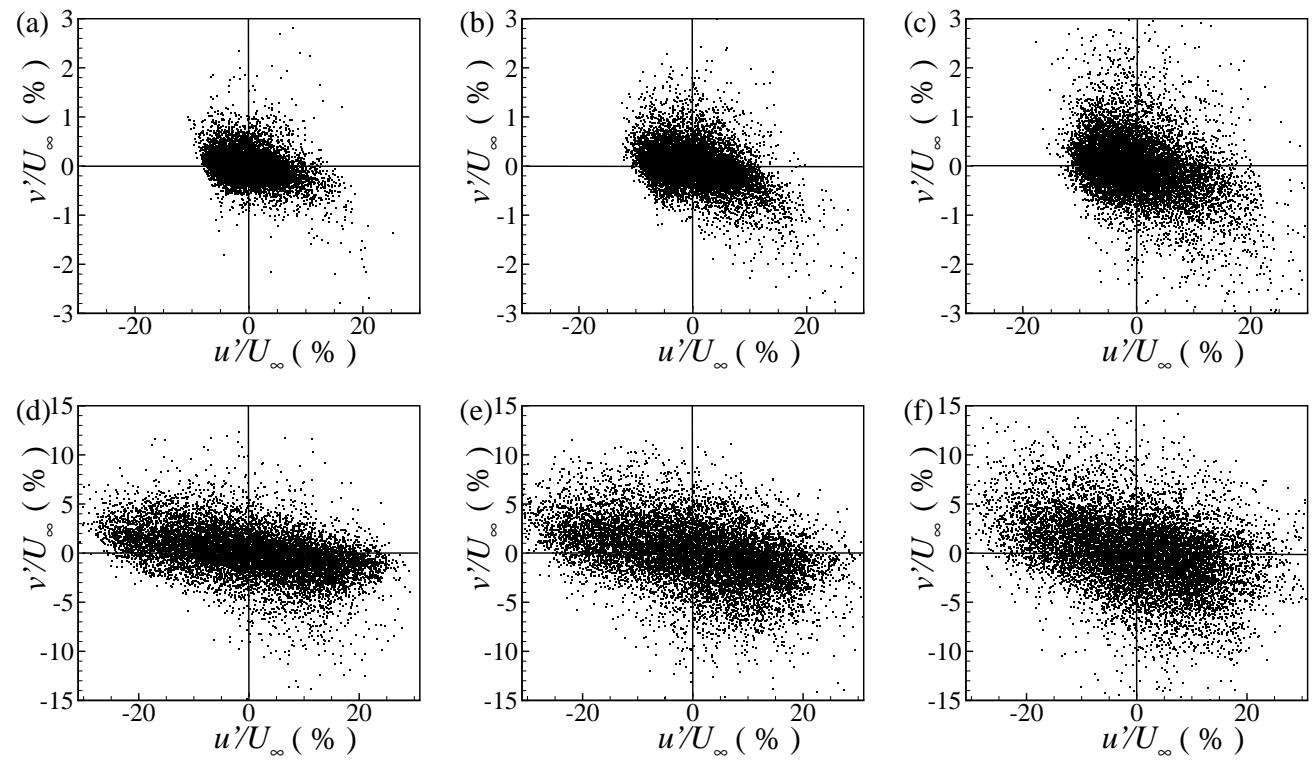

Figure 31. Scatter plots of the Reynolds stress quadrant decomposition. (a-c) At the wall-normal position of $y / \delta_{i}=0.008$ in the viscous sub-layer; (d-f) at the wall-normal position of $y / \delta_{i}=0.04$ in the buffer layer. (a,d) At the streamwise position of $x=18.3 \delta_{i}$; (b,e) at the streamwise position of $\alpha=3.0^{\circ} ;(\mathrm{c}, \mathrm{f})$ at the streamwise position of $\alpha=9.0^{\circ}$.

The profiles of averaged $u^{\prime} v^{\prime}$ normalized by $U_{\infty}^{2}$ for the four quadrants at $x=18.3 \delta_{i}$ in the flat region are given in figure32(a). For all four quadrants of the flat-plate boundary layer, $(u v)_{i} / U_{\infty}^{2}$ peaks around the wall-normal position of $y / \delta_{i}=0.1$ (corresponding to $\left.y^{+}=32\right)$. Negative values of the Q2 and Q4 events are much larger than those of the Q1 and Q3 events. The respective contribution of each quadrant could be further evaluated by normalizing the averaged $u^{\prime} v^{\prime}$ with total Reynolds shear stress $(u v)_{a v e}$. The profiles are given in figure 32(b). Obviously, Q2 and Q4 events contribute predominantly to the mean Reynolds shear stress throughout the boundary layer. In the near-wall region, the Q4 event contributes the majority of Reynolds stress, indicating that the near-wall turbulence is mainly generated by the sweep event. Its contribution decreases fast with the wall-normal height in the region from the wall to $y / \delta_{i}=0.1$, while simultaneously, the contribution of Q2 increases quickly, taking the most important role in the Reynolds shear stress. Further away from the wall, while the importance of Q4 event in the Reynolds shear stress being gradually weakened with the wall-normal height, the importance of Q2 event is gradually promoted.

Considering the importance of Q2 and Q4 events, profiles of the quadrant decomposed Q2 and Q4 Reynolds stress at five streamwise positions are given in figure 33. Obviously, both Q2 ejection and Q4 sweep events are significantly strengthened in the concave boundary layer. Both the ejection and sweep events are correlated with the wall-normal motions. The strengthened ejection and sweep events are the direct results of the largescale roll cells which induce the high-momentum flow to move inward while induce the low-momentum flow to move outward at the same time. The strengthening of ejection and sweep events noted here is also responsible for the streamwise increase of Reynolds shear stress that have been pointed in Section 3.2. While the Reynolds shear stress is closely related to the turbulent production, the turbulent intensity is promoted. In addition, it also can be found in figure 33 that along with the streamwise increase of 

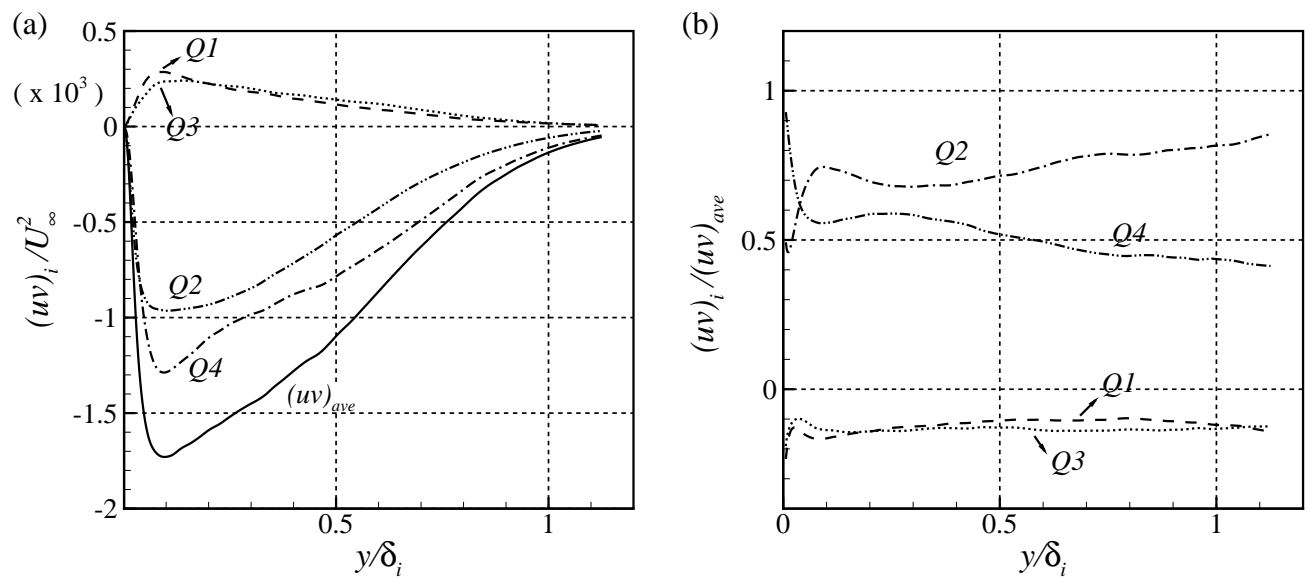

Figure 32. Quadrant decomposition analysis of the Reynolds stress at the streamwise position of $x=18.3 \delta_{i}$ in the flat plate region. (a) Profiles of quadrant decomposed Reynolds stress; (b) contributions of different events to the mean Reynolds stress.
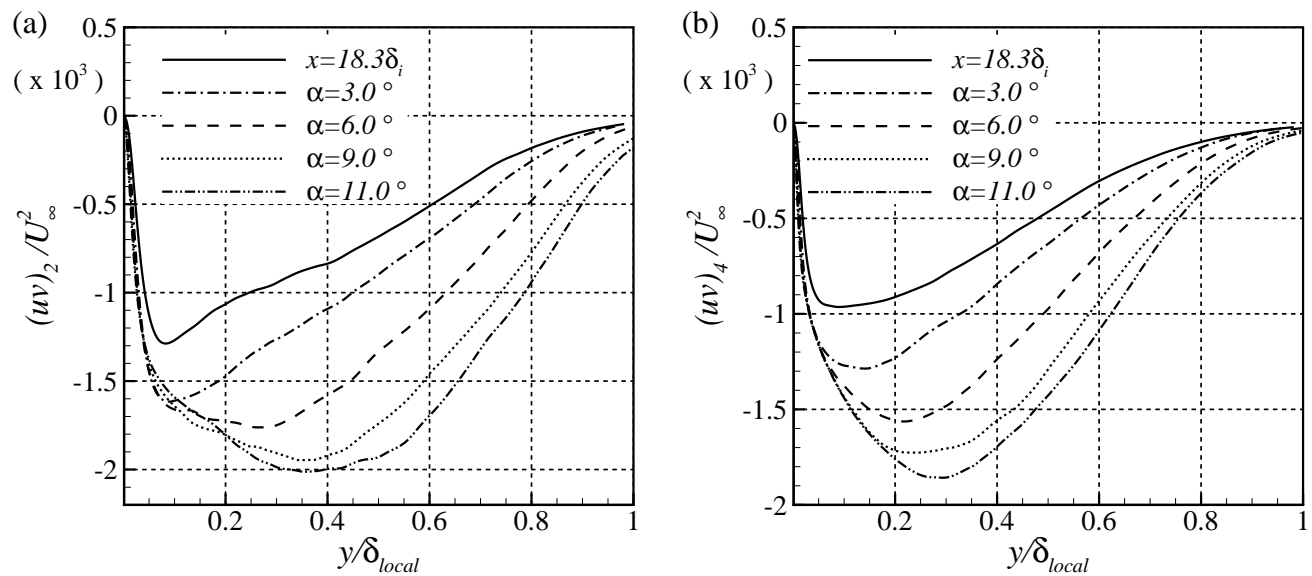

Figure 33. Profiles of quadrant decomposed Reynolds stress at different streamwise positions.

(a) $Q 2$ event; (b) $Q 4$ event

sweep and ejection events, their peaking positions are gradually moving outwards as well. This suggests a higher turbulent production in the outer layer in the supersonic concave turbulent boundary layer. As a result, second bumps are introduced in the profiles for the streamwise and spanwise turbulent intensity. For the same reason, peak positions in profiles of the wall-normal turbulent intensity and the Reynolds shear stress move outwards with the wall turning as well.

To further identify the boundary layer events in the $x z$ plane, scatter plots of the quadrant decomposition for $u^{\prime}$ and $w^{\prime}$ at two wall-normal positions which are $y / \delta_{i}=0.008$ in the viscous sub-layer and $y / \delta_{i}=0.228$ in the logarithmic layer are given in figure 34 , where $w^{\prime}$ is the spanwise velocity fluctuation. At each wall-normal position, scatter plots at three streamwise positions which are $x=18.3 \delta_{i}, \alpha=3.0^{\circ}$ and $\alpha=9.0^{\circ}$ are given. It can be found in the figure that at both wall-normal positions the spanwise fluctuation $w^{\prime}$ tends to have larger value with the wall turning. The promotion of spanwise fluctuation in the concave boundary layer is consistent with our findings in Section 3.3.1 where large- 

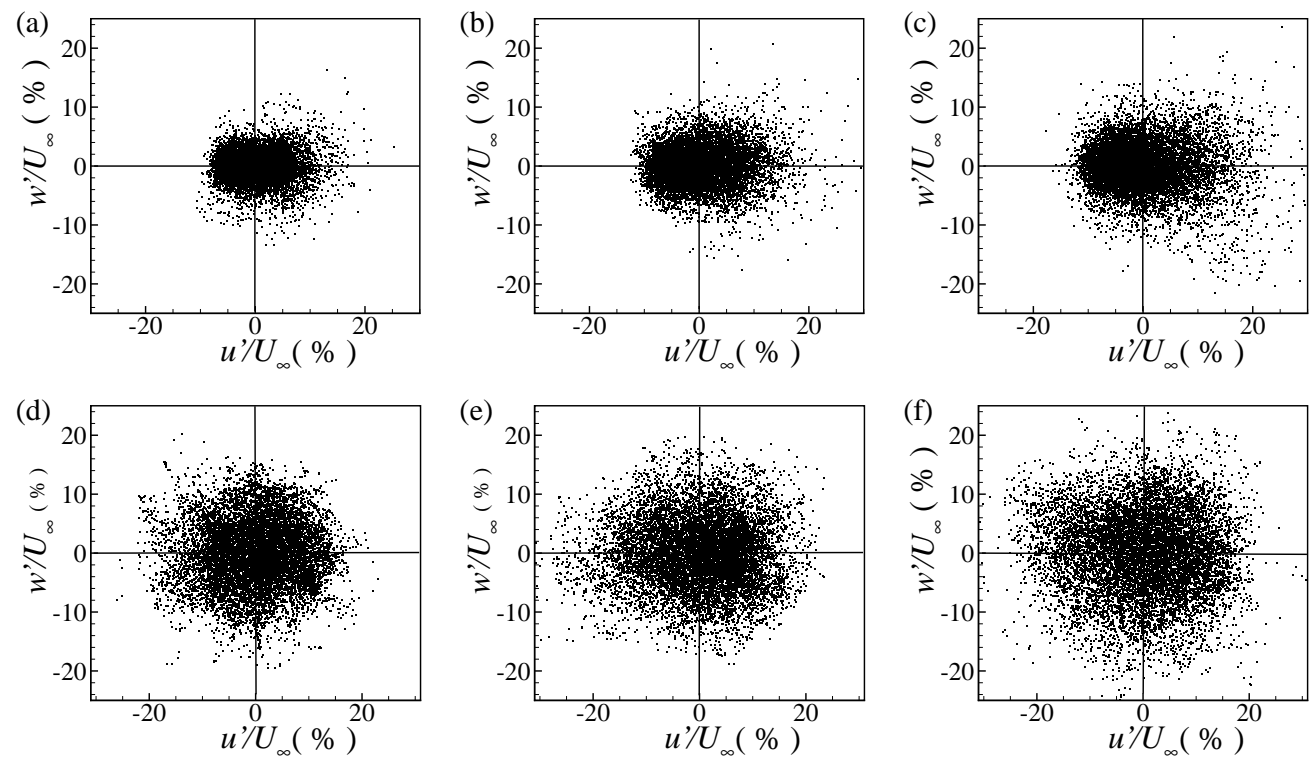

Figure 34. Scatter plots of the quadrant decomposition in the $x z$ plane. (a-c) At the wall-normal position of $y / \delta_{i}=0.008$ in the viscous sub-layer; (d-f) at the wall-normal position of $y / \delta_{i}=0.228$ in the logarithmic layer. (a,d) At the streamwise position of $x=18.3 \delta_{i}$; $(\mathrm{b}, \mathrm{e})$ at the streamwise position of $\alpha=3.0^{\circ} ;(\mathrm{c}, \mathrm{f})$ at the streamwise position of $\alpha=9.0^{\circ}$.

scale longitudinal roll cells are identified. This also explains the streamwise increase of spanwise turbulent intensity.

\subsubsection{Two-point spatial correlation}

To check the response of large-scale coherent motions to the streamwise concave curvature, two-point spatial correlation maps for the streamwise velocity component are calculated in the wall-parallel and $x y$ planes. In the wall-parallel plane, the spatial correlation is calculated with the relation:

$$
R_{u u}\left(x_{0}+\Delta x, y_{0}, \Delta z\right)=\frac{\overline{u^{\prime}\left(x_{0}, y_{0}\right) \cdot u^{\prime}\left(x_{0}+\Delta x, y_{0}, \Delta z\right)}}{\sqrt{\overline{u^{\prime}\left(x_{0}, y_{0}\right)^{2}}} \cdot \sqrt{\overline{u^{\prime}\left(x_{0}+\Delta x, y_{0}\right)^{2}}}}
$$

where, the subscript ' 0 ' denotes the reference position, $\Delta x$ and $\Delta z$ are the in-plane streamwise and spanwise separations, respectively. The correlation results in the wallparallel planes with $y^{+}=10$ and $y^{+}=100\left(y^{+}\right.$is calculated using the friction velocity $u_{\tau}$ at the reference streamwise position) are given in figures 35 and 36, respectively. In each plane, the results at four reference streamwise positions are presented, which are $x=18.3 \delta_{i}, \alpha=3.0^{\circ}, \alpha=6.0^{\circ}$ and $\alpha=9.0^{\circ}$.

Obviously, the iso-contours are streamwise extended, which is a typical feature of the correlation map in the wall-parallel plane. It reflects the existence of low-speed streaks, but its streamwise dimension does not strictly reveal the streamwise length of the streak due to its meandering feature (Hutchins \& Marusic 2007). Clearly, the streamwise correlation varies with the wall turning at both wall-normal positions. In the plane of $y^{+}=10$ (see figure 35), the streamwise correlation increases from $x=18.3 \delta_{i}$ in the flat-plate region to $\alpha=6.0^{\circ}$ in the concave region. Further downstream to $\alpha=9.0^{\circ}$, it is evidently decreased. This is mainly attributed to the large number of high-momentum patches induced by the large-scale roll cells from the outer layer(see figure 30(a) in the 

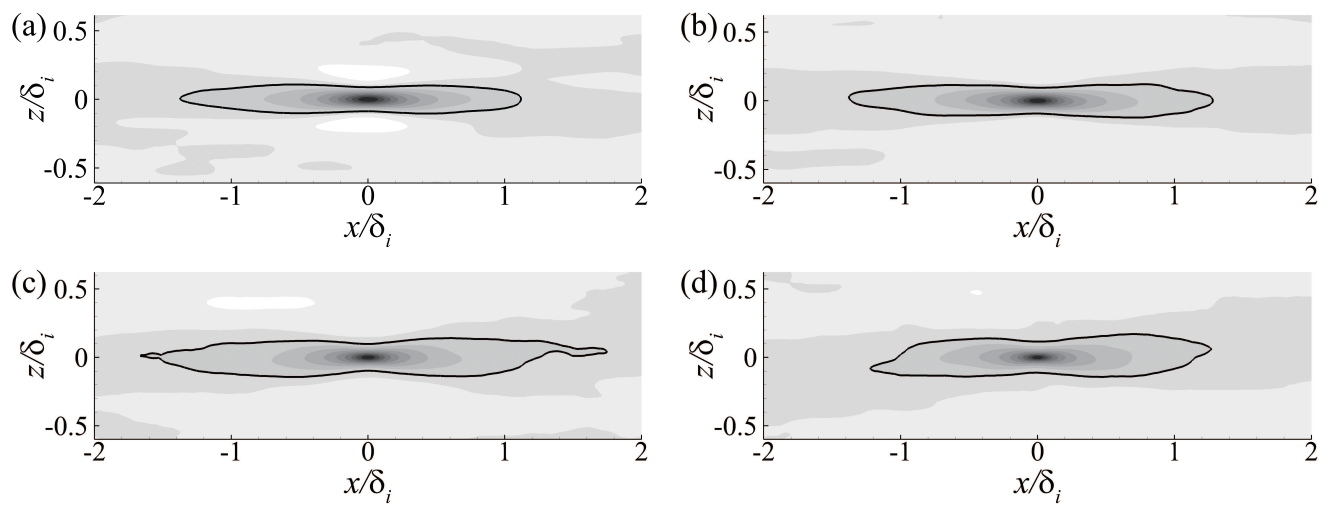

Figure 35. Two-point spatial correlation maps in the wall-parallel plane of $y^{+}=10$. Twelve levels between -0.1 and 1.0 with increment of 0.1 are shown. The black solid contour line plotted is for the correlation value of 0.1 . Results are acquired at four reference streamwise positions: (a) $x=18.3 \delta_{i}$, (b) $\alpha=3.0^{\circ}$, (c) $\alpha=6.0^{\circ}$, (d) $\alpha=9.0^{\circ}$.
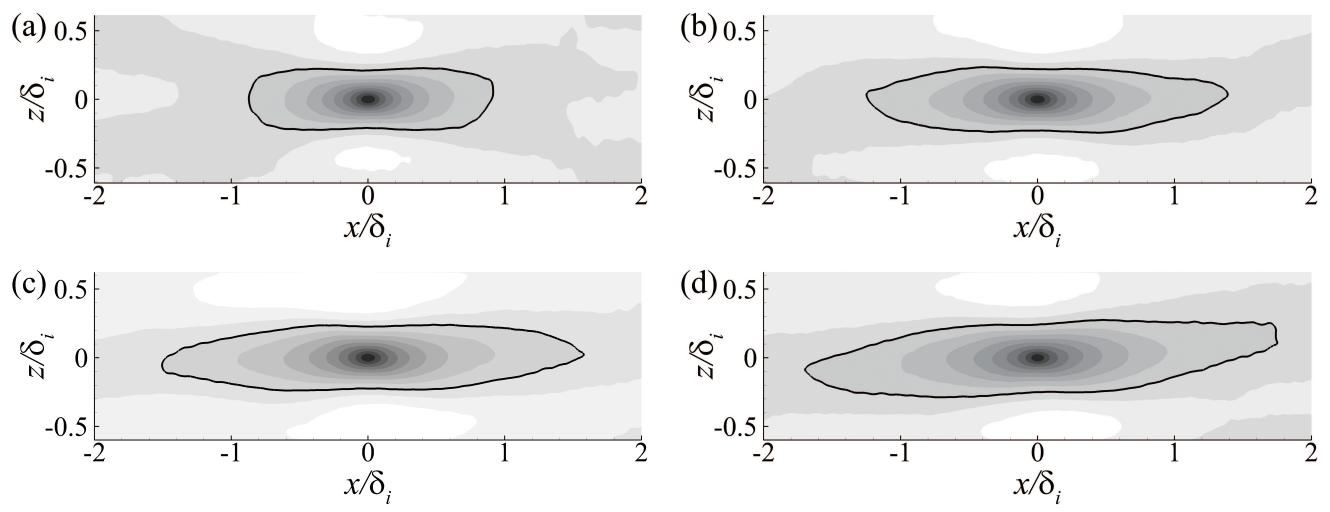

Figure 36. Two-point spatial correlation maps in the wall-parallel plane of $y^{+}=100$. Twelve levels between -0.1 and 1.0 with increment of 0.1 are shown. The black solid contour line plotted is for the correlation value of 0.1 . Results are acquired at different streamwise reference positions: (a) $x=18.3 \delta_{i}$, (b) $\alpha=3.0^{\circ}$, (c) $\alpha=6.0^{\circ}$, (d) $\alpha=9.0^{\circ}$.

region from $x / \delta_{i}=27$ to 31 ), which dominantly truncate the near-wall streaks and diminish the streamwise correlation in the concave region. At the higher wall-parallel plane with $y^{+}=100$, the streamwise dimension of the iso-contour at $x=18.3 \delta_{i}$ is obviously smaller than that at $y^{+}=10$. However, the length of the iso-contour increases persistently in the streamwise direction. At the streamwise position of $\alpha=9.0^{\circ}$, the streamwise correlation of the turbulence in the plane of $y^{+}=100$ is much higher than that in the plane of $y^{+}=10$. The higher streamwise correlation at $y^{+}=100$ is mainly attributed to the large-scale roll cells which make the turbulence more streamwise correlated in the low wake region.

In addition to the variation of streamwise dimension of the iso-contour, the spanwise dimension is also found to vary both in the streamwise and wall-normal directions, as shown in figures 35 and 36. To have a clearer evaluation of the iso-contour width, the spanwise profiles of two-point correlation at five streamwise reference positions are extracted. Similar to that given in figures 35 and 36, the correlation results are acquired at two wall-normal positions which are $y^{+}=10$ and $y^{+}=100$, as shown in figure 37 . 

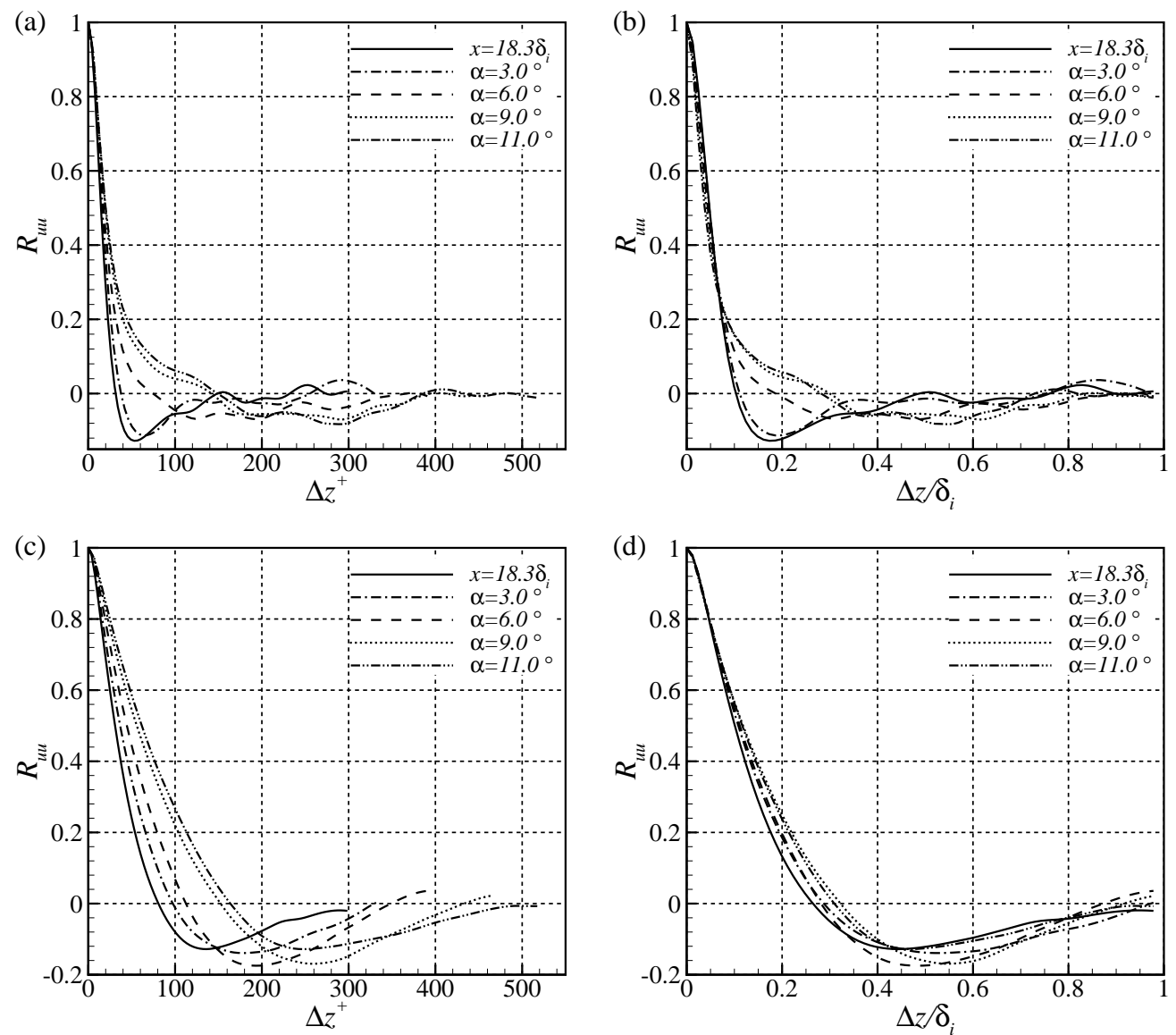

FiguRE 37. Spanwise profiles of two-point spatial correlation $R_{u u}$ at different streamwise positions. The results are presented for various wall-normal positions: $(\mathrm{a}, \mathrm{b}) y^{+}=10 ;(\mathrm{c}, \mathrm{d})$ $y^{+}=100$. Different normalizing ways are used for the spanwise width: (a,c) the spanwise width is denoted by $z^{+} ;(\mathrm{b}, \mathrm{d})$ the spanwise width is denoted by $z / \delta_{i}$

Two ways of normalization for the spanwise dimension are employed. In figure 37 (a) and (c), the width is denoted by $z^{+}$, and in figure 37 (b) and (d), the width is normalized with the inflow boundary layer thickness $\delta_{i}$ to present the absolute value.

With inner scaling, the iso-contour width denoted by $z^{+}$is evidently increased with the wall turning in both wall-parallel planes, as given in figure 37 (a) and (c). The absolute value of width is also found to increase in the streamwise direction in the wall-parallel plane with $y^{+}=10$. But in the higher plane, its streamwise variation is relatively weak. Thus the noted streamwise growth of the iso-contour width in figure 37(c) is mainly attributed to the streamwise decrease of friction velocity.

Two-point spatial correlation maps in the $x y$ plane are also calculated by using the relation

$$
R_{u u}\left(x_{0}+\Delta x, y_{0}+\Delta y\right)=\frac{\overline{u^{\prime}\left(x_{0}, y_{0}\right) \cdot u^{\prime}\left(x_{0}+\Delta x, y_{0}+\Delta y\right)}}{\sqrt{\overline{u^{\prime}\left(x_{0}, y_{0}\right)^{2}}} \cdot \sqrt{\overline{u^{\prime}\left(x_{0}+\Delta x, y_{0}+\Delta y\right)^{2}}}}
$$

The correlations at two wall-normal reference positions which are $y^{+}=5$ and $y^{+}=50$ 
34
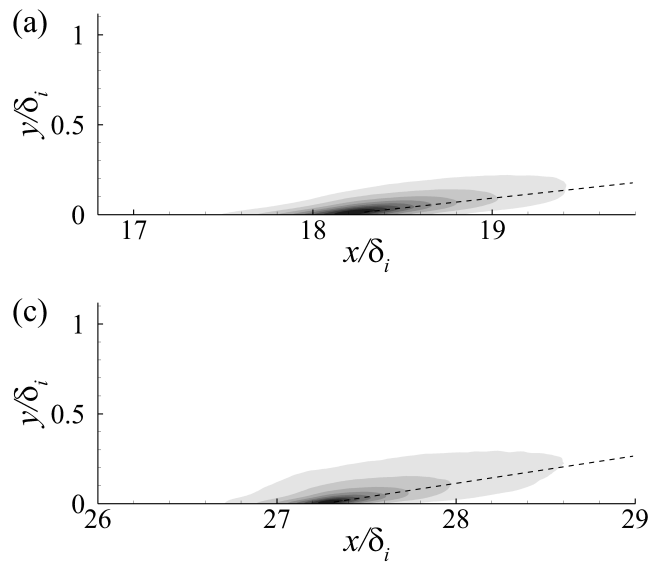

Q. C. Wang and others
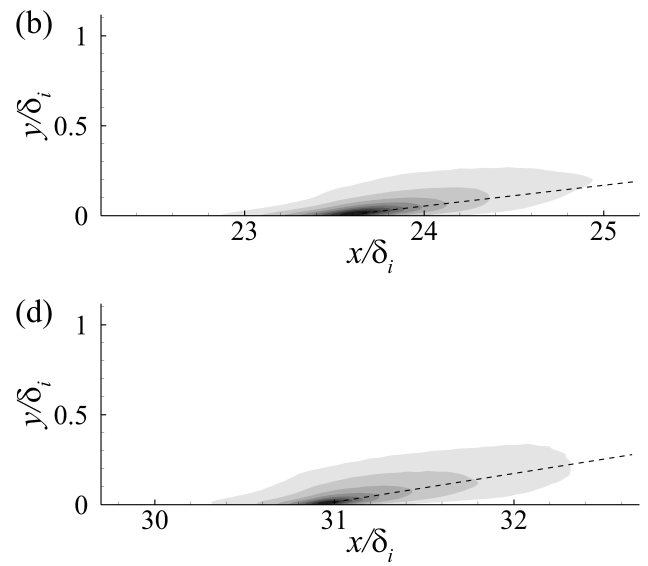

FiguRE 38. Two-point spatial correlation maps in the XY plane at the wall-normal reference position of $y^{+}=5$. The streamwise reference position varies: (a) $x=18.3 \delta_{i}$, (b) $\alpha=3.0^{\circ}$, (c) $\alpha=6.0^{\circ},(\mathrm{d}) \alpha=9.0^{\circ}$. The outer (minimum) correlation value is 0.2 .
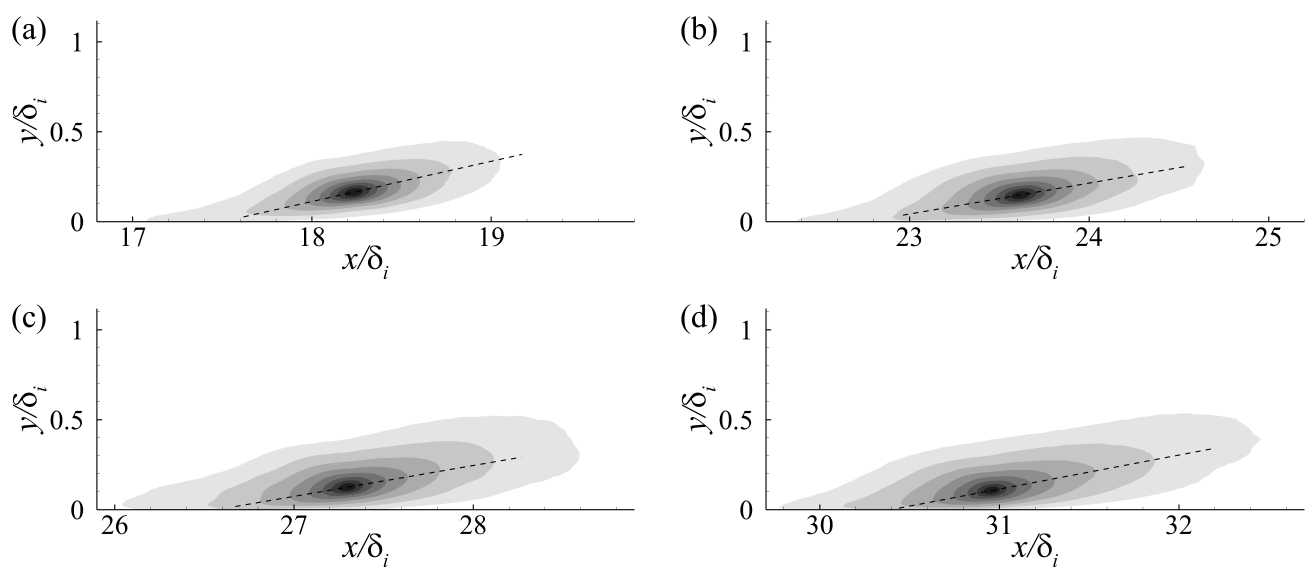

FiguRE 39. Two-point spatial correlation maps in the XY plane at the wall-normal reference position of $y^{+}=50$. The streamwise reference position varies: (a) $x=18.3 \delta_{i}$, (b) $\alpha=3.0^{\circ}$, (c) $\alpha=6.0^{\circ},(\mathrm{d}) \alpha=9.0^{\circ}$. The outer (minimum) correlation value is 0.2 .

are calculated, as given in figures 38 and 39. At each wall-normal position, results at four streamwise positions are presented.

It is clear that all of the iso-contours are seen to possess a forward-leaning elliptical distribution, which is consistent with previous studies regarding turbulent boundary layers (Tichenor et al. 2013; Wang et al. 2016a; Humble et al. 2012). At the higher wall-normal position, the iso-contours are found to lean farther away from the wall. Since the leaning angle of an iso-correlation map represents the growth angle of a hairpin packet, which is defined by the envelope of the within hairpin heads (Adrian et al. 2000; Wang et al. 2017), the variation of the leaning angle indicates that the growth angle of the hairpin packet at $y^{+}=50$ is larger that at $y^{+}=10$. In addition, the non-dimensional spatial extent of the iso-contour appears to be larger at the higher wall-normal position, suggesting larger, higher-correlated turbulent structures are present in the outer region of the boundary layer.

With the wall turning, spatial extents of the iso-contours are also found to increase at 
both wall-normal reference positions, indicating that the streamwise concave curvature can give higher-correlated turbulent structures. In figure 38, it can be clearly seen that the wall-normal dimension of the iso-contour increases with the wall turning. Since the reference position is at $y^{+}=5$, which is located in the near-wall region, the observed increase of wall-normal dimension suggests that the near-wall turbulent structures tend to be more correlated with the outer structures in the concave turbulent boundary layer. The streamwise increase of the correlation value in the near-wall region noted in figure 39 also supports this.

The physical cause of this can be found in figure 23 where the streamwise change of instantaneous turbulent structures is presented. As can be noted and has been pointed out in figure 23(b), in the near-wall region of the flat-plate turbulent boundary layer, the quasi-streamwise vortices dominate. Due to their streamwise extending feature, their leaning angles to the wall usually tend to be very small and therefore the wall-normal correlations decrease quickly. The direct result of this is the narrow iso-contour observed in figure 38(a) and the small correlation value noted in the near-wall region in figure 39(a). In the concave boundary layer, more hairpin vortices are generated in the region close to the wall, as shown in figure 23(c). Considering their shapes, the hairpin vortices usually tend to tilt and curved away from the wall, which could evidently improve the wall-normal correlation. Thus, the considerable number of hairpin vortices generated in the near-wall region in the concave turbulent layer are the main cause of the streamwise increase of wall-normal size of the iso-contour noted in figure 38 and the increased correlation value in the near-wall region found in figure 39.

\section{Discussion and conclusion}

In this paper, the response of a Mach 2.95 turbulent boundary layer to the streamwise concave curvature has been investigated in detail by using DNS. A method to define the boundary layer thickness physically based on the principal strain rate is proposed, which is applicable for boundary layer subjected to wall-normal pressure and velocity gradients. The DNS results presented in Sections 3.1 and 3.2 have shown that both the mean properties and the turbulent statistics are significantly affected by the streamwise concave curvature.

Turbulent structures are significantly altered by the concave curvature due to the Görtler instability. Streamwise extended vortices are intensified and more vortices are generated. Accumulations of these vortices lead to the formation of the large-scale streamwise roll cells. Similar to the incompressible flow, the existence of the large-scale streamwise roll cells is also a dominant feature in the supersonic concave boundary layer. The streamwise concave curvature can amplify the incoming spanwise non-uniformities. For a turbulent boundary layer, if no spanwise stationary perturbations are deliberately imposed and the incoming flow is free from the resulted spanwise non-uniformities, the large-scale roll cells are originated from the VLSMs. The wall-normal-motion related events (ejection and sweep) are promoted by the roll cells. Both the inward and outward motions are strengthened by the roll cells, through which the high-momentum flow are found to have a higher tendency to move towards the wall, while the low-momentum flow tends to move towards the outer edge.

The streamwise alterations of mean properties and turbulent statistics in the supersonic concave boundary layer are closely related to the formation of large-scale streamwise roll cells. Because of the strengthened inward motion brought by the roll cells, the mean velocity in the near-wall region is increased in the region of $y / \delta_{\text {local }}<0.03$. For the same reason, the wall friction and the principal strain in the near-wall region (Layer I, 
$\left.y / \delta_{\text {local }}<0.012\right)$ are increased as well. The large number of high-speed patches noticed in the concave region is the direct result of the strengthened inward motion. Considering the decrease of velocity at the edge of the boundary layer due to the bulk compression, the streamwise increase of the near-wall velocity magnitude must be compensated in outer regions. This is achieved by the strengthened outward motion which moves the low-momentum flow in the inner layer to the outer edge, leading to the decrease of streamwise velocity in the outer layer. Because of this, the velocity gradient is smoothed, the principal strain is decreased (Layer II, $0.015<y / \delta_{\text {local }}<0.2$ ) and the slope of the logarithmic region is reduced as well. Higher in the region $0.4<y / \delta_{\text {local }}<0.8$ (Layer III), the principal strain has been very small. In Layer III, the mean velocity is close to the free-stream. Due to the strengthened ejection, the low momentum is induced to move to the outer edge of the boundary layer to slightly promote the velocity gradient in the wallnormal direction around the boundary layer edge. As a consequence, the principal strain is increased. According to the physical model given by Wang et al. (2016b) to depict the interaction between the compression wave and the hairpin packet, the compression waves may contribute to the streamwise increase of principal strain too.

The large-scale roll cells promote the interaction between the low-momentum and the high-momentum flow throughout the boundary layer. Most significant promotion of the interaction occurs in the outer layer. As a result, the turbulent level is increased. Due to the significant promotion of the sweep and ejection events in the outer layer, secondary bumps for the streamwise and spanwise turbulent intensity are found at the wall-normal height around $y / \delta_{\text {local }} \approx 0.3$. Due to the same reason, peak positions of wall-normal fluctuation and Reynolds shear stress are also found to move outward to the height around $y / \delta_{\text {local }} \approx 0.3$. Along with the promotion of turbulent level by the roll cells, the more small-scale vortices are generated and large amount of hairpin vortices are found to be formed in the near-wall region, which leads to stronger wall-normal correlation.

\section{Acknowledgement}

The authors gratefully acknowledge Prof. N. D. Sandham for his valuable support. This work is funded by the National Science Foundation of China (Grants: 11472304, 11472305 and 11522222).

\section{REFERENCES}

Adrian, R. J., Meinhart, C. D. \& Tomkins, C. D. 2000 Vortex organization in the outer region of the turbulent boundary layer. J. Fluid Mech. 422, 1-54.

BARlow, R. S. \& Johnston, J. P. 1988 a Local effects of large-scale eddies on bursting in a concave boundary layer. J. Fluid Mech. 191, 177-195.

Barlow, R. S. \& Johnston, J. P. $1988 b$ Structure of a turbulent boundary layer on a concave surface. J. Fluid Mech. 191, 137-176.

Bradshaw, P. 1973 Effects of streamline curvature on turbulent flow. AGARDograph 169.

Bradshaw, P. 1974 The effect of mean compression or dilatation on the turbulence structure of supersonic boundary layers. J. Fluid Mech. 63, 449-464.

Chakraborty, P., Balachandar, S. \& Adrian, R. J. 2005 On the relationships between local vortex identification schemes. J. Fluid Mech. 535, 189-214.

Chong, M. S., Perry, A. E. \& Cantwell, B. J. 1990 A general classification of threedimentional flow fields. Phys. Fluids 2, 765-777.

Clauser, F. H. 1954 Turbulent boundary layers in adverse pressure gradients. J. Aeronaut. Sci. 21, 91-108.

Dempsey, L. J., Hall, P. \& Deguchi, K. 2017 The excitation of Görtler vortices by free stream coherent structures. J. Fluid Mech. 826, 60-96. 
Donovan, J. F., Spina, E. F. \& Smits, A. J. 1994 The structure of a supersonic turbulent boundary layer subjected to concave surface curvature. J. Fluid Mech. 259, 1-24.

Elsinga, G. E., Adrian, R. J., Oudheusden, B. W. Van \& Scarano, F. 2010 Threedimensional vortex organization in a high-reynolds-number supersonic turbulent boundary layer. J. Fluid Mech. 644, 35-60.

Fernholzh, H. H. \& Finley, P. J. 1980 A critical commentary on mean flow data for two dimensional compressible turbulent boundary layers. AGARDograph 253. North Atlantic Treaty Organization.

Flaherty, W. \& Austin, J. M. 2013 Scaling of heat transfer augmentation due to mechanical distortions in hypervelocity boundary layers. Phys. Fluids 25, 106106.

Floryan, J. M. 1991 On the Görtler instability of boundary layers. Prog. Aerospace Sci. 28, $235-271$.

Franko, K. J. \& Lele, S. 2014 Effect of adverse pressure gradient on high speed boundary layer transition. Phys. Fluids 26, 024106.

GöRTLER, H. 1954 On the three dimensional instability of laminar boundary layers on concave walls. NACA TM 1375.

Guarini, S. E., Moser, R. D., Shariff, K. \& Wray, A. 2000 Direct numerical simulation of a supersonic turbulent boundary layer at mach 2.5. J. Fluid Mech. 414, 1-33.

HALL, P. 1983 The linear development of Görtler vortices in growing boundary layers. J. Fluid Mech. 130, 41-58.

Harun, Z., Mont, J. P., Mathis, R. \& Marusic, I. 2013 Pressure gradient effects on the large-scale structure of turbulent boundary layers. J. Fluid Mech. 715, 477-498.

Hoffmann, P. H., Muck, K. C. \& Bradshaw, P. 1985 The effect of concave surface curvature on turbulent boundary layers. J. Fluid Mech. 161, 371-403.

Hopkins, E. J. \& InOuye, M. 1971 An evaluation of theories for predicting turbulent skin friction and heat transfer on flat plates at supersonic and hypersonic mach numbers. AIAA J. 9, 993-1003.

Humble, R. A., Peltier, S. J. \& Bowersox, R. D. W. 2012 Visualization of the structural response of a hypersonic turbulent boundary layer to convex curvature. Phys. Fluids 24, 106103.

Hutchins, N. \& Marusic, I. 2007 Evidence of very long meandering features in the logarithmic region of turbulent boundary layers. J. Fluid Mech. 579, 1-28.

Hutchins, N., Nickels, T. B., Marusic, I. \& Chong, M. S. 2009 Hot-wire spatial resolution issues in wall-bounded turbulence. J. Fluid Mech. 635, 103-136.

Jayaram, M., TAYlor, M. W. \& Smits, A . J. 1987 The response of a compressible turbulent boundary layer to short regions of concave surface curvature. J. Fluid Mech. 175, 343-362.

Kim, J., Moin, P. \& Moser, R. 1987 Turbulence statistics in fully developed channel flow at low reynolds number. J. Fluid Mech. 177, 133-166.

Kim, K. C. \& Adrian, R. J. 1999 Very large-scale motion in the outer layer. Phys. Fluids 11, $417-423$.

LADERMAN, A. J. 1980 Adverse pressure gradient effects on supersonic boundary layer turbulence. AIAA J. 18 (10).

LeE, J. H. \& Sung, H. J. 2011 Very-large-scale motions in a turbulent boundary layer. J. Fluid Mech. 673, 80-120.

Lewis, J. E., Gran, R. L. \& Kubota, T. 1972 An experiment on the adiabatic compressible turbulent boundary layer in adverse and favourable pressure gradients. J. Fluid Mech. 51, 657-672.

Lu, S. S. \& Willmarth, W. W. 1973 Measurements of the structure of the reynolds stress in a turbulent boundary layer. J. Fluid Mech. 60 (3), 481-511.

Maeder, T., Adams, N. A. \& Kleiser, L. 2001 Direct simulation of turbulent supersonic boundary layers by an extended temporal approach. J. Fluid Mech. 429, 187-216.

Marusic, I., Mathis, R. \& Hutchins, N. 2010 High reynolds number effects in wall turbulence. Int. J. Heat and Fluid Flow 31, 418-428.

Mokhtarzadeh-Dehghan, M. R. \& Yuan, Y. M. 2002 Measurements of turbulence quantities and bursting period in developing turbulent boundary layers on the concave and convex walls of a $90^{\circ}$ square bend. Exp. Thermal Fluid Sci. 27, 59-75. 
Monty, J. P., Harun, Z. \& Marusic, I. 2011 A parametric study of adverse pressure gradient turbulent boundary layers. Int. J. Heat and Fluid Flow 32, 575-585.

Ozalp, A. A. \& UmuR, H. 2003 An experimental investigation of the combined effects of surface curvature and streamwise pressure gradients both in laminar and turbulent flows. Heat and Mass Transfer 39, 869-876.

Patel, V. C. \& Sotiropoulos, F. 1997 Longitudinal curvature effects in turbulent boundary layers. Prog. Aerospace Sci. 33, 1-70.

Pirozzoli, S., Grasso, F. \& Gatski, T. B. 2004 Direct numerical simulation and analysis of a spatially evolving supersonic turbulent boundary layer at $\mathrm{m}=2.25$. Phys. Fluids 16, $530-545$.

Pokrajac, D., Campbell, L. J., Nikora, V., Manes, C. \& McEwan, I. 2007 Quadrant analysis of persistent spatial velocity perturbations over square-bar roughness. Exp Fluids 42, 413-423.

Ren, J. \& Fu, S. 2015 Secondary instabilities of Görtler vortices in high-speed boundary layer flows. J. Fluid Mech. 781, 388-421.

Ringuette, M. J., Wu, M. \& Martin, M. P. 2008 Coherent structures in direct numerical simulation of turbulent boundary layers at mach 3. J. Fluid Mech. 594, 59-69.

Roghelia, A., Olivier, H., Egorov, I. \& Chuvakhov, P. 2017 Experimental investigation of Görtler vortices in hypersonic ramp flows. Exp Fluids 58 (10).

SANDhAM, N. D. 2016 Effects of compressibility and shock-wave interactions on turbulent shear flows. Flow Turbul. Combust. 97, 1-25.

Sandham, N. D., LI, Q. \& Yee, H. C. 2002 Entropy splitting for high-order numercial simulation of compressible turbulence. J. Comput. Phys. 178, 307-322.

Sandham, N. D., Schlein, E., Wagner, A., Willems, S. \& Steelant, J. 2014 Transitional shock-wave/boundary-layer interactions in hypersonic flow. J. Fluid Mech. 752, 349-382.

Saric, W. S. 1994 Görtler vortices. Annu. Rev. Fluid Mech. 26, 379-409.

Schlatter, P. \& Orlu, R. 2010 Assessment of direct numerical simulation data of turbulent boundary layers. J. Fluid. Mech. 659, 116-126.

Schrader, L., Brandt, L. \& ZAKI, T. A. 2011 Receptivity, instability and breakdown of Görtler flow. J. Fluid Mech. 682, 362-396.

Smith, C. R. \& Metzler, S. P. 1983 The characteristics of low-speed streaks in the near-wall region of a turbulent boundary layer. J. Fluid Mech. 129, 27-54.

Smith, D. R. \& Smits, A. J. 1995 A study of the effects of curvature and a supersonic turbulent boundary layer. Exp Fluids 18, 363-369.

Smits, A. J., McKeon, B. J. \& Marusic, I. 2011 High-reynolds number wall turbulence. Ann. Re. Fluid Mech. 43, 353-375.

Smits, A. J. \& Wood, D. H. 1985 The response of turbulent boundary layers to sudden pertubations. Ann. Re. Fluid Mech. 17, 321-358.

Spalding, D. B. 1961 A single formula for the law of the wall. J. Appl. Mech. 28, 455-457.

Sturek, W. B. \& Danberg, J. E. $1972 a$ Supersonic turbulent boundary layer in adverse pressure gradient, part 2: data analysis. AIAA J. 10 (5).

Sturek, W. B. \& Danberg, J. E. $1972 b$ Supersonic turbulent boundary layer in adverse pressure gradient, part i: the experiment. AIAA J. 10 (4).

Sun, M. B., Hu, Z. \& Sandham, N. D. 2017 Recovery of a supersonic turbulent boundary layer after an expansion corner. Phys. Fluids 29, 076103.

Swearingen, J. D. \& Blackwelder, R. F. 1987 The growth and breakdown of streamwise vortices in the presence of a wall. J. Fluid Mech. 182, 255-290.

Tandiono, T., Winoto, S. H. \& Sнан, D. A. 2008 On the linear and nonlinear development of Görtler vortices. Phys. Fluids 20, 094103.

Tichenor, N. R., Humble, R. A. \& Bowersox, R. D. W. 2013 Response of a hypersonic turbulent boundary layer to favourable pressure gradients. J. Fluid Mech. 722, 187-213.

Tong, F., Li, X., DuAn, Y. \& YU, C. 2017 Direct numerical simulation of supersonic turbulent boundary layer subjected to a curved compression ramp. Phys. Fluids 29, 125101.

Touber, E. 2010 Unsteadiness in shock wave boundary layer interactions. PhD thesis, University of Southampton.

Touber, E. \& Sandham, N. D. 2009 Large-eddy simulation of low-frequency unsteadiness in a turbulent shock-induced separation bubble. Theor. Comput. Fluid Dyn. 23, 79-107. 
VINOKUR, M. 1980 On one-dimensional stretching functions for finite-difference calculations. NASA Contractor Report 3313.

Wang, B., Sandham, N. D., Hu, Z. \& W, Liu. 2015b Numerical study of oblique shockwave/boundary-layer interaction considering sidewall effect. J. Fluid Mech. 767, 526-561.

Wang, J. S., Feng, L. H., WAng, J. J. \& LI, T. 2018 Görtler vortices in low-reynolds-number flow over multi-element airfoil. J. Fluid Mech. 835, 898-935.

WANG, Q. C. \& WANG, Z. G. 2016 Structural characteristics of the supersonic turbulent boundary layer subjected to concave curvature. Appl. Phys. Lett. 108, 114102.

WANG, Q. C., WANG, Z. G. \& ZhaO, Y. X. 2016a An experimental investigation of the supersonic turbulent boundary layer subjected to concave curvature. Phys. Fluids 28, 096104.

Wang, Q. C., Wang, Z. G. \& ZhaO, Y. X. $2016 b$ On the impact of adverse pressure gradient on the supersonic turbulent boundary layer. Phys. Fluids 28, 116101.

Wang, Q. C., WAng, Z. G. \& ZhaO, Y. X. 2017 The impact of streamwise convex curvature on the supersonic turbulent boundary layer. Phys. Fluids 29, 116106.

White, F. M. 2006 Viscous Fluid Flow, 3rd edn. McGraw-Hill.

Wu, X., Zhaо, D. \& Luo, J. 2011 Excitation of steady and unsteady Görtler vortices by free-stream vortical disturbances. J. Fluid Mech. 682, 66-100.

Wu, Y. \& Christensen, K. T. 2006 Population trends of spanwise vortices in wall turbulence. J. Fluid Mech. 568, 55-76.

Xie, Z. T. \& CAStro, I. P. 2008 Efficient generation of inflow conditions for large-eddy simulation of street-scale flows. Flow Turbul. Combust. 81, 449-470.

Yee, H. C., Vinokur, M. \& Djomenri, M. J. 2000 Entropy splitting and numerical dissipation. J. Comput. Phys. 162, 33-81.

Zhou, J., Adrian, R. J., Balachandar, S. \& Kendall, T. M. 1999 Mechanisms for generating coherent packets of hairpin vortices in channel flow. J. Fluid Mech. 387, 353396. 